\title{
Reflexionen von Multiplikatorinnen und Multiplikatoren zum Gestaltungsprinzip der Teilnehmendenorientierung - Fachspezifische Professionalisierung beim Design von Fortbildungen
}

\author{
Petra Scherer • Marcus Nührenbörger $\cdot$ Leonie Ratte
}

Eingegangen: 30. September 2018 / Angenommen: 18. November 2020 / Online publiziert: 14. Dezember 2020

(C) Der/die Autor(en) 2020, korrigierte Publikation 2021

Zusammenfassung In diesem Beitrag werden erste Ergebnisse einer qualitativen Studie vorgestellt, die Reflexionen von Multiplikatorinnen und Multiplikatoren zur allgemeinen Konzeption und zur konkreten Durchführung eines Fortbildungskurses mit dem Thema „Inklusiver Mathematikunterricht“ für fachfremde, aber im inklusiven Mathematikunterricht der Grundschule tätige Sonderpädagoginnen und Sonderpädagogen untersucht. Dazu hospitierten vier Multiplikatorinnen einerseits bei einem existierenden Kurs, andererseits reflektierten sie diesen mit Blick auf eine zukünftig eigenständige Durchführung des Kurses. Im Beitrag werden die Reflexionen der Multiplikatorinnen zum Gestaltungsprinzip der Teilnehmendenorientierung näher analysiert. Die Ergebnisse der Analysen von schriftlichen Beobachtungsprotokollen und moderierten Reflexionsgesprächen weisen auf normative und prospektive Reflexionen von Multiplikatorinnen und Multiplikatoren hin.

Schlüsselwörter Fortbildung · Professionalisierung · Multiplikatorinnen und Multiplikatoren · Fachfremder Mathematikunterricht · Inklusion

MESC-Codes $\mathrm{B} 50 \cdot \mathrm{B} 60 \cdot \mathrm{C} 90$

Petra Scherer $(\bowtie) \cdot$ Leonie Ratte

Fakultät für Mathematik, Campus Essen, Universität Duisburg-Essen,

Thea-Leymann-Straße 9, 45127 Essen, Deutschland

E-Mail: petra.scherer@uni-due.de

Marcus Nührenbörger

Fakultät für Mathematik/IEEM, Technische Universität Dortmund,

Vogelpothsweg 87, 44227 Dortmund, Deutschland 


\title{
Facilitators' Reflections on the Design Principle of Participant Orientation - Content-Specific Professionalization in the Design of In- Service Courses
}

\begin{abstract}
This paper presents first results of a qualitative study that investigates facilitators' reflections concerning an in-service course "Inclusive mathematics education" and its concept for special education teachers who can be classified as outof-field teachers but at the same time teach mathematics in inclusive settings. On the one hand, four facilitators sit in on an existing course, on the other hand they reflect the course concept in perspective to a future independent performing of the course. In the paper the facilitators' reflections on the design principle of participant orientation are analyzed. The analyses of written feedbacks and reflective discussions indicate facilitators' normative as well as prospective reflections.
\end{abstract}

Keywords In-service teacher education · Professionalization · Facilitators · Outof-field teaching $\cdot$ Inclusion

\section{Motivation und Zielsetzung}

Spätestens seit der Ratifizierung der UN-Behindertenrechtskonvention (BRK) und dem Ausbau inklusiver Schulen sind eine Vielzahl von Fragen zur Gestaltung des inklusiven Unterrichts und zur Professionalisierung der Lehrpersonen aufgekommen. So hat die Notwendigkeit einer Erweiterung der sonderpädagogischen Expertise an allgemeinbildenden Schulen zu einem vermehrten Einsatz fachfremd unterrichtender Sonderpädagoginnen und Sonderpädagogen im inklusiven Fachunterricht der Regelschulen geführt (vgl. Heinrich et al. 2013, S. 71f). Da für das professionelle Handeln im inklusiven Fachunterricht neben sonderpädagogischen Kompetenzen und grundlegenden Überzeugungen auch fachliche und fachdidaktische Wissensbestände von Interesse sind (vgl. Heinrich et al. 2013; Korff 2015; Gasterstädt und Urban 2016), kommen der Planung und Durchführung von Fortbildungen ${ }^{1}$, in denen Sonderpädagoginnen und Sonderpädagogen entsprechende fachliche und fachdidaktische Grundlagen des inklusiven Mathematikunterrichts erwerben können, besondere Bedeutung zu. Wie Fortbildende, im Folgenden als Multiplikatorinnen und Multiplikatoren bezeichnet, bei der Planung und Durchführung entsprechender Maßnahmen vorgehen oder wie sie bei der Bewältigung dieser Aufgaben unterstützt werden können, ist bisher allerdings wenig erforscht. Diese Forschungsdesiderate aufgreifend ist es das Ziel des Beitrags, die Reflexionen von Multiplikatorinnen und Multiplikatoren bei der Beobachtung und der anschließenden Planung einer

\footnotetext{
1 Da die Begriffe „Fortbildung“ und „Weiterbildung“ innerhalb des deutschen Sprachraums keine einheitliche Verwendung finden (vgl. u.a. Reusser und Tremp 2008), soll im Folgenden auf eine differenzierte Nutzung der beiden Termini verzichtet werden. Damit schließt der hier verwendete Begriff der ,Fortbildung“ nicht nur die Weiterentwicklung bereits in der Ausbildungsphase erworbener professioneller Kompetenzen ein, sondern auch den Aufbau neuer berufsbezogener Kompetenzen, wie sie beispielsweise fachfremd unterrichtende Lehrpersonen benötigen.
} 
entsprechenden Fortbildungsmaßnahme mit Blick auf das Gestaltungsprinzip der Teilnehmendenorientierung zu untersuchen.

\section{Theoretische Rahmung}

\subsection{Professionelle Kompetenzen von Multiplikatorinnen und Multiplikatoren}

Als Multiplikatorinnen und Multiplikatoren werden Personen bezeichnet, die Lehrpersonen ,fortbilden, beraten und deren Unterrichtsentwicklung sowie Bildungsund Erziehungsarbeit unterstützen und begleiten“ (Barzel und Selter 2015, S. 262; vgl. auch Schuler und Wittmann 2018). Für den Bereich der Fortbildung haben Lipowsky und Rzejak (2017) ein Angebots-Nutzungs-Modell entwickelt (vgl. auch Lipowsky 2014), welches die in einer Fortbildung angebotenen Lerngelegenheiten sowie deren Wahrnehmung und Nutzung durch die teilnehmenden Lehrpersonen in den Mittelpunkt der Betrachtung stellt. Dabei sind Qualität und Quantität entsprechender Lerngelegenheiten - wie auch der Fortbildungsmaßnahme insgesamt auf die professionellen Kompetenzen beziehungsweise Merkmale der verantwortlichen Multiplikatorinnen und Multiplikatoren, also ihr professionelles Wissen, ihre professionellen Überzeugungen und ihre Motivationsfähigkeit, zurückzuführen. Das professionelle Wissen von Multiplikatorinnen und Multiplikatoren umfasst neben fortbildungsmethodischen Elementen, wie etwa dem Wissen um fortbildungsbezogene Gestaltungsprinzipien ${ }^{2}$, insbesondere auch fachliche und fachdidaktische Wissensinhalte und bezieht diese Bereiche aufeinander. Allerdings existieren bislang keine einheitlichen Standards und nur wenige Konzepte zur Qualifizierung von Multiplikatorinnen und Multiplikatoren (vgl. Rösken-Winter und Kramer 2013, S. 842; Lipowsky und Rzejak 2017, S. 392).

Die zentrale Bedeutung der professionellen Kompetenzen von Multiplikatorinnen und Multiplikatoren für die Wirksamkeit von Fortbildungsangeboten (vgl. Borko et al. 2014; Lipowsky und Rzejak 2017) berücksichtigend wurde im Rahmen des Deutschen Zentrums für Lehrerbildung Mathematik (DZLM) das vom Deutschen Institut für Erwachsenenbildung (DIE) entwickelte GRETA ${ }^{3}$-Kompetenzmodell - welches sich auf den Bereich der allgemeinen Erwachsenenbildung bezieht (Lencer und Strauch 2016) - für das Fach Mathematik adaptiert (vgl. Peters-Dasdemir et al. 2020). Dabei wurden existierende mathematikspezifische Überlegungen zu den professionellen Kompetenzen von Multiplikatorinnen und Multiplikatoren aufgegriffen (u. a. Zaslavsky 2008; Borko et al. 2014).

\footnotetext{
2 Für fachbezogene Fortbildungen im Bereich Mathematik hat das Deutsche Zentrum für Lehrerbildung Mathematik (DZLM) anknüpfend an verschiedene Forschungsarbeiten (vgl. z. B. Wilson und Berne 1999; Timperley et al. 2007) sechs zentrale Gestaltungsprinzipien herausgearbeitet: Kompetenzorientierung, Teilnehmendenorientierung, Lehr-Lern-Vielfalt, Fallbezug, Kooperationsanregung und Reflexionsförderung (vgl. Barzel und Selter 2015; Rösike et al. 2016; Barzel et al. 2018).

3 Bei GRETA handelt es sich um ein vom Bundesministerium für Bildung und Forschung (BMBF) gefördertes Projekt, dessen Ziel die Schaffung von „Grundlagen für die Entwicklung eines trägerübergreifenden Anerkennungsverfahrens für die Kompetenzen Lehrender in der Erwachsenenbildung und Weiterbildung“ (GRETA) ist (vgl. Lencer und Strauch 2016, S. 2).
} 
Abb. 1 Kompetenzmodell für Mathematikmultiplikatorinnen und -multiplikatoren des DZLM (Peters-Dasdemir et al. 2020)

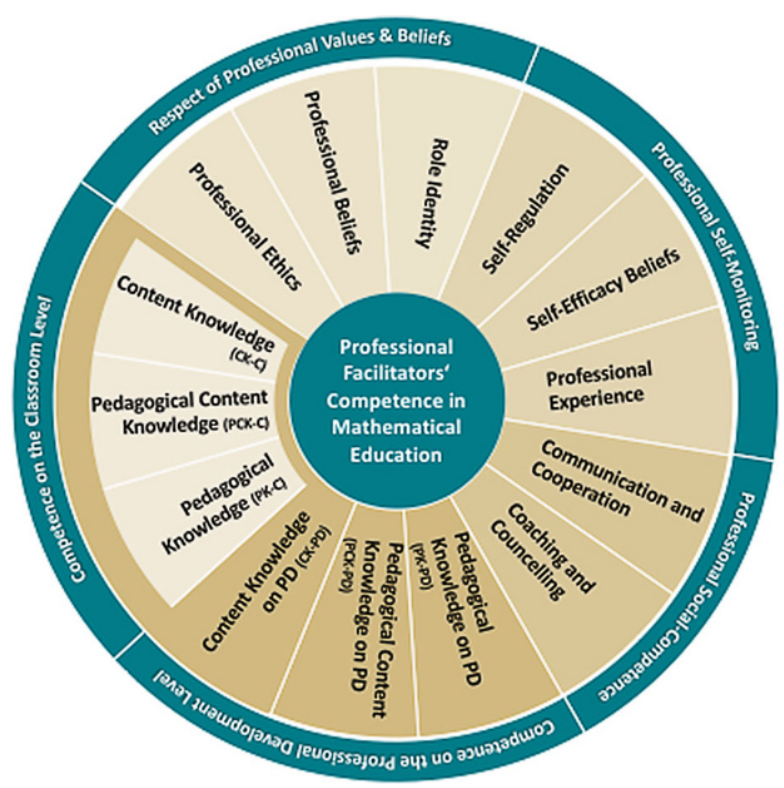

Das Modell (vgl. Abb. 1), das im Folgenden genauer erläutert werden soll, bildet das Wissen der Mathematikmultiplikatorinnen und -multiplikatoren auf der Fortbildungsebene ab und umfasst ihr Fortbildungsgegenstandswissen (Content Knowledge on Professional Development; kurz: PD), ihr fachdidaktisches Fortbildungswissen (Pedagogical Content Knowledge on PD) und ihr pädagogisches Wissen in Bezug auf die Bildung Erwachsener (Pedagogical Knowledge on PD). Das Fortbildungsgegenstandswissen beinhaltet das Wissen auf der Unterrichtsebene - also Fachwissen (Content Knowledge), fachdidaktisches Wissen (Pedagogical Content Knowledge) und allgemein pädagogisches Wissen (Pedagogical Knowledge), wie es beispielsweise in dem von Baumert und Kunter (2006) erarbeiteten Modell professioneller Handlungskompetenzen von Lehrpersonen herausgestellt wird (vgl. Abschn. 2.3) und geht in Bezug auf den Fortbildungsgegenstand über dieses Wissen hinaus. Neben den beschriebenen kognitiven Wissensbereichen bezieht das Modell die professionellen Werthaltungen und Überzeugungen (Professional Ethics, Professional Beliefs and Role Identity), die Fähigkeit zur professionellen Selbststeuerung (SelfRegulation, Self-Efficacy Beliefs and Professional Experience) und die professionelle Sozialkompetenz (Communication and Cooperation, Coaching and Councelling) von Multiplikatorinnen und Multiplikatoren mit ein, da diese Kompetenzfacetten die Handlungen von Multiplikatorinnen und Multiplikatoren entscheidend beeinflussen dürften.

\subsection{Reflexion als zentrale Komponente professionellen Handelns}

Grundsätzlich ist davon auszugehen, dass das professionelle Handeln von Mathematikmultiplikatorinnen und -multiplikatoren und dessen Weiterentwicklung ähnlich wie bei Lehrpersonen auf Reflexionen angewiesen sind. In der Lehrerbildung gilt 
es als unbestritten, dass die Reflexion eine entscheidende Rolle bei der Kompetenzentwicklung von Lehrpersonen einnimmt und eine wichtige Komponente ihres professionellen Handels darstellt (vgl. u. a. Herzog 1995; Wyss 2008; Dalehefte und Kobarg 2013).

Neuweg (2005, S. 15) definiert Professionalität von Lehrpersonen in diesem Sinne als das „Wechselspiel von Einlassung auf Erfahrung, Reflexion auf Erfahrung und Rückübersetzung in neues Handeln. Professionell ist ein Lehrer in dieser Perspektive dann weder auf Grund seines Wissens noch auf Grund des schlichten Ausmaßes seiner Erfahrung, sondern erst, wenn er zudem einen analytischen Habitus ausgeprägt hat, also bereit ist, Handlungspraxis regelmäßig zu analysieren, zu evaluieren und gegebenenfalls zu verändern.“" Obwohl sich in der Literatur eine Vielzahl unterschiedlicher Reflexionskonzepte finden lässt (vgl. Schön 1988; Herzog 1995; Wyss 2008, 2013), geht der Grundgedanke eines erfahrungsbasierten beziehungsweise reflexiven Lernens im Wesentlichen auf Dewey zurück (vgl. Cendon 2017, S. 40). Dewey (2000, S. 195) fasst als Reflexion „denkende Handlungen“ auf, die im Zusammenhang mit der Suche nach Problemlösungen und deren Überprüfungen stehen (vgl. auch Dewey 2002, S. 13). Unterschieden wird bei Reflexionen zwischen der „Reflexion-in-der-Handlung“ (reflection-in-action) und der „Reflexionüber-die-Handlung“" (reflection-on-action; vgl. Schön 1983). Ziele des nachgelagerten Reflexionsprozesses sind die kritische Prüfung des eigenen Verhaltens und die Entwicklung neuer (Handlungs-)Strategien für zukünftige Handlungsanforderungen (vgl. Cendon 2017, S. 40). Folglich kann eine weitere Unterscheidung zwischen retrospektiver und prospektiver Reflexion (vgl. Herzog 1995) festgehalten werden, also dem Rückblick auf vollzogene Handlungen oder aber die Perspektive auf zukünftige Handlungen und damit verbundene Adaptionsüberlegungen. Prospektive Reflexion umfasst in diesem Sinne auch die von Sherin und Drake (2009, S. 486f) herausgestellten Adaptionsprozesse von Lehrpersonen, die eine Nutzung bestehender Materialien konkretisieren (vgl. auch Leufer et al. 2019): Es wird etwas weggelassen (omit) oder ersetzt (replace), oder es wird etwas Neues geschaffen (create).

Eine Möglichkeit, Reflexionsprozesse bei Lehrpersonen anzuregen, ist die kollegiale Hospitation, welche auf eine konstruktive Rückmeldung an die hospitierte Person ausgerichtet ist (vgl. Buhren 2012, S. 8). Anders verhält es sich im Falle von Unterrichtsbeobachtungen. Hier sollen Verstehens- und Reflexionsprozesse auf Seiten der beobachtenden Person angeregt werden (vgl. Bennewitz 2012, S. 212), um sie in ihrer professionellen Entwicklung zu unterstützen. Somit können Reflexionen sowohl auf das eigene unterrichtliche Handeln ausgerichtet sein (Selbstreflexion) als auch im Sinne einer Fremdreflexion auf das Handeln anderer Lehrpersonen (vgl. Wyss 2008). Nicht selten wird zudem zwischen geschlossener (gemäß definierter Kriterien) und offener (gemäß selbst gewählter Kriterien) Reflexion differenziert (vgl. Wyss 2008).

Für das Lehren von Mathematik weisen beispielsweise Scherer und Steinbring (2006) explizit auf die Bedeutung einer kritisch-distanzierten Beobachtung von Unterricht und auf die reflexive Kommunikation von Lehrpersonen über die eigenen Lehrpraktiken hin, um letztlich professionelle Bewusstheit zu entwickeln (vgl. auch Nührenbörger und Steinbring 2009; Berndt et al. 2017). Allerdings zeigen Forschungsergebnisse, dass Bereitschaft und Fähigkeit zur Reflexion bei Lehrpersonen 
wenig ausgeprägt sind (vgl. Wyss 2008, S. 12). Erst mit zunehmender Erfahrung sind höhere Reflexionsprozesse zu erwarten (vgl. Sato et al. 1993). Vor diesem Hintergrund stellt sich die Frage, inwiefern sich diese Erkenntnisse auf Ebene der Lehrpersonen auf die Ebene von Multiplikatorinnen und Multiplikatoren, die in der Regel über große Berufserfahrung verfügen, übertragen lassen.

In der hier berichteten Studie soll daher der Fragestellung nachgegangen werden, welche Bedeutung die Reflexion auf der Ebene der Multiplikatorinnen und Multiplikatoren hat (vgl. Abschn. 5.1 und 5.2). Zum einen wird untersucht, wie Multiplikatorinnen und Multiplikatoren retrospektiv das Handeln anderer Akteure im Rahmen einer Fortbildungshospitation reflektieren. Zum anderen wird genauer beleuchtet, wie sie prospektiv auf das eigene Handeln hinsichtlich einer zukünftig eigenständigen Durchführung dieser Fortbildung reflektieren. Da die Wirkung und damit auch der Erfolg von Fortbildungsmaßnahmen in besonderer Weise auch durch die an einer Maßnahme teilnehmenden Lehrpersonen beeinflusst werden, wie Angebots-Nutzungs-Modelle aufzeigen (z. B. Lipowsky und Rzejak 2017), ist für die Reflexionsprozesse der Multiplikatorinnen und Multiplikatoren das Gestaltungsprinzip der Teilnehmendenorientierung von besonderer Bedeutung.

\subsection{Zum Gestaltungsprinzip der Teilnehmendenorientierung}

Teilnehmende einer Fortbildung weisen unterschiedliche kognitive und motivationale Voraussetzungen auf, die ausschlaggebend dafür sind, in welcher Weise sie die angebotenen Lerngelegenheiten wahrnehmen und nutzen können. Hinzu kommen verschiedene persönlichkeitsbezogene und (berufs-)biographische Voraussetzungen sowie unterschiedliche schulische Kontextbedingungen, die einen Einfluss auf die Wirksamkeit entsprechender Angebote nehmen dürften (vgl. Lipowsky 2010, S. 51 f; Lipowsky und Rzejak 2017, S. 381). Das DZLM-Gestaltungsprinzip der Teilnehmendenorientierung ist auf die Erhebung und den Einbezug von „Vorwissen, Vorerfahrungen, Erwartungen und Bedürfnisse[n]“ (Barzel und Selter 2015, S. 271) der Teilnehmenden einer Fortbildung ausgerichtet. Damit Lehrpersonen ihre professionellen Kompetenzen im Rahmen von Fortbildungsmaßnahmen anpassen und weiterentwickeln können, ist es wichtig, dass ihnen die Gelegenheit gegeben wird, bereits vorhandenes Wissen aktiv einzubringen (vgl. Carpenter und Fennema 1992; Clarke 1994; Krainer 1998; Franke et al. 2001; Borasi und Fonzi 2002), um auf dieser Grundlage neue Kompetenzen und Perspektiven zu entwickeln (vgl. DZLM 2015, S. 7).

Hinsichtlich der kognitiven Voraussetzungen von Lehrpersonen unterscheiden Baumert und Kunter (2006, S. 482) zwischen ihrem allgemeinpädagogischen, ihrem fachlichen und fachdidaktischen Wissen sowie ihrem Organisations- und Beratungswissen. In Bezug auf die motivationalen Voraussetzungen wird zwischen „Überzeugungen und Werthaltungen“, ,motivationalen Orientierungen“ und „,selbstregulativen Fähigkeiten“ von Lehrpersonen unterschieden. Des Weiteren beeinflussen die Beweggründe für die Fortbildungsteilnahme (siehe hierzu Rzejak et al. 2014) und die Orientierung an den individuellen Bedarfen und Interessen der Teilnehmenden die Erfolgsaussichten von Fortbildungen (z. B. Franke et al. 2001; Krainer 1998). Auch wenn die Akzeptanz und die Zufriedenheit von Lehrpersonen nicht automatisch zu 
einer nachhaltigen Veränderung des professionellen Wissens führen, dürften sie sich doch zumindest indirekt ,-- über das Engagement und die Intensität der Nutzung der Fortbildungsangebote durch die Lehrer/innen - auf den Lern- und Transferprozess auswirken“ (Lipowsky 2010, S. 54). Neben den persönlichen Voraussetzungen der Lehrpersonen nimmt auch deren schulischer Kontext einen Einfluss darauf, inwieweit sich Fortbildungsmaßnahmen als wirksam erweisen können (Lipowsky und Rzejak 2017). Das Gestaltungsprinzip der Teilnehmendenorientierung umfasst daher auch die Vereinbarkeit der in einer Fortbildung behandelten Themen mit den Zielen und Inhalten des Schulprogramms der Lehrpersonen ebenso wie die Berücksichtigung des spezifischen Klassenkontextes und der Schülerschaft.

\subsection{Fachfremd unterrichtende Sonderpädagoginnen und Sonderpädagogen im Fokus}

Mit Blick auf die Teilnehmendengruppe der fachfremd unterrichtenden Sonderpädagoginnen und Sonderpädagogen sind hinsichtlich des Professionswissens einige Besonderheiten zu berücksichtigen. Moser und Kropp (2015, S. 189) unterscheiden sieben spezifische Kompetenzbereiche des Professionswissens von Sonderpädagoginnen und Sonderpädagogen: „Lernstands- und Entwicklungsdiagnostik“, „Beratungs- und Organisationskompetenz“, „Binnendifferenzierte Unterrichtung“, ,Lernund Entwicklungsförderung“, „Behinderungsspezifische Kommunikation“, „Interdisziplinäre Kooperation“ und „Förderung des sozialen Lernens“. Die verschiedenen Kompetenzbereiche weisen zwar einen Bezug zu dem von Baumert und Kunter (2006) beschriebenen allgemeinpädagogischen Professionswissen sowie dem Organisations- und Beratungswissen von Lehrpersonen auf, gleichwohl deuten sie auf spezifische Aspekte des Professionswissens der Teilnehmendengruppe hin, die bei Regelschullehrpersonen in dieser Ausprägung nicht vorliegen: Die Sonderpädagoginnen und Sonderpädagogen verfügen vor allem über eine besondere förderpädagogische Expertise, die besondere Diagnosen und Förderkonzepte bei einzelnen Lernenden umfasst. Eine effektive sonderpädagogische Förderung von Schülerinnen und Schülern setzt jedoch nicht nur die Berücksichtigung des zugrundeliegenden Entwicklungsschwerpunkts voraus (vgl. Wember 1998, S. 108), sondern verlangt darüber hinaus nach einer fachspezifischen Diagnose, welche auf der Basis fachlichen und fachdidaktischen Wissens erfolgen muss (vgl. Baumert und Kunter 2006, S. 489; Moser Opitz und Nührenbörger 2015, S. 497).

In der Fortbildungsmaßnahme, die der vorliegenden Studie zugrunde liegt, zeichnet sich die Teilnehmendengruppe der fachfremd unterrichtenden Sonderpädagoginnen und Sonderpädagogen also durch mindestens zwei Spezifika aus, welche es bei der Planung und Durchführung einer Fortbildungsmaßnahme zum inklusiven Mathematikunterricht zu berücksichtigen gilt: Auf der einen Seite verfügen die Teilnehmenden über keine mathematische beziehungsweise mathematikdidaktische Ausbildung, was jedoch nicht bedeuten muss, dass keine praktischen Erfahrungen in diesen Bereichen vorliegen. Auf der anderen Seite bringen die Teilnehmenden eine besondere Expertise im Bereich der Sonderpädagogik mit, welche die entsprechende Expertise vieler Mathematikmultiplikatorinnen und -multiplikatoren übersteigen dürfte. Darüber hinaus unterscheidet sich der schulische Einsatz zahlreicher Sonder- 
pädagoginnen und Sonderpädagogen deutlich von dem der meisten Regelschullehrpersonen.

\subsection{Das Drei-Tetraeder-Modell der gegenstandsbezogenen Professionalisierungsforschung}

In Anlehnung an das klassische didaktische Dreieck hat das DZLM ein Drei-Tetraeder-Modell entwickelt, welches die Ebene des Unterrichts mit den Ebenen der Lehrerfortbildung und der Multiplikatorinnen- und Multiplikatorenqualifizierung verknüpft (vgl. Abb. 2). Die Eckpunkte des didaktischen Dreiecks, welche durch die Lehrenden, die Lernenden und den zu erlernenden Gegenstand gebildet werden, wurden hierzu um den Eckpunkt der verwendeten Medien und Materialien ergänzt und anschließend von der Unterrichts- zunächst auf die Fortbildungs- und schließlich auf die Qualifizierungsebene übertragen (Prediger et al. 2017, S. $161 \mathrm{ff}$ ).

Die rechte Seitenfläche des jeweiligen Tetraeders verbindet die handelnden Akteure und - je nach Ebene - den fachlichen Lern-, Fortbildungs- oder Qualifizierungsgegenstand, indem sie nach gegenstandsspezifischen Lernunterstützungen der Lehrenden für die Lernenden fragt. Die Strukturierung des jeweiligen Gegenstands und seine didaktische Aufbereitung durch den Einsatz von Medien und Material werden auf den linken Seitenflächen abgebildet und die gegenstandsspezifischen Lernwege und die kognitiven Aktivitäten der Lernenden auf den unteren Seitenflächen. Die hinteren Seitenflächen nehmen schließlich gegenstandsunabhängige pädagogische Methoden der Gestaltung von Lehr-Lernumgebungen in den Blick (vgl. Prediger et al. 2017, S. 162).

\section{Qualifizierungsebene}

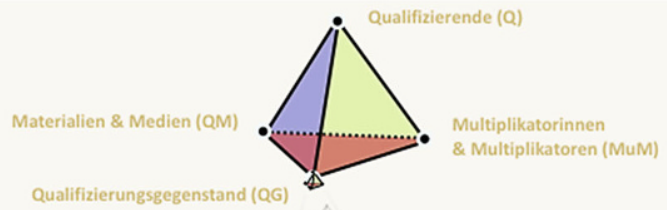

Fortbildungsebene

(Qualifizierungsgegenstand)

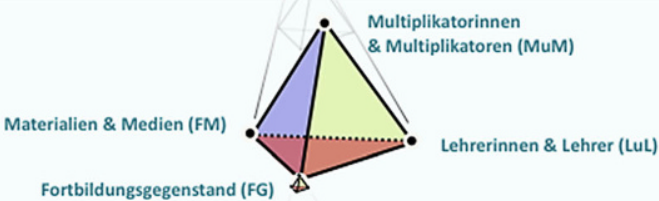

Unterrichtsebene

(Fortbildungsgegenstand)

Tetraederseiten

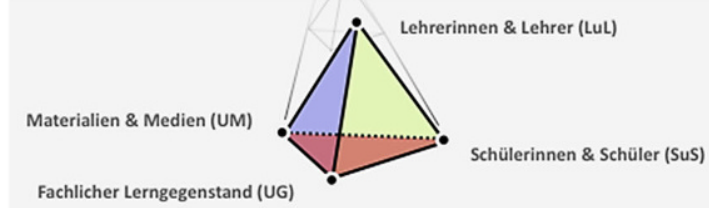

Gegenstandsspezifische Lernunterstützung

Strukturierung des Gegenstands und mater
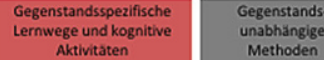

unabhàngige

Abb. 2 Drei-Tetraeder-Modell der gegenstandsbezogenen Professionalisierungsforschung des DZLM (in Anlehnung an Prediger et al. 2017) 
Fortbildungsebene

(Qualifizierungsgegenstand)

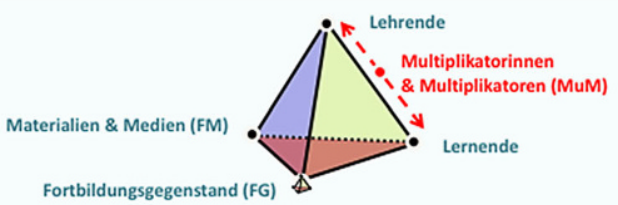

Abb. 3 Rolle von Multiplikatorinnen und Multiplikatoren im Drei-Tetraeder-Modell während einer Fortbildungshospitation

Während den Lehrpersonen auf der Unterrichtsebene die Rolle der Lehrenden zukommt, werden sie auf der Fortbildungsebene zu Lernenden, deren Lernprozesse durch die in der Lehrerfortbildung tätigen Multiplikatorinnen und Multiplikatoren initiiert und begleitet werden. Fortbildungsgegenstand ist dabei - der Grundidee einer Schachtelung der Lerngegenstände nach Luft und Hewson (2014) folgend nicht allein der fachliche Lerngegenstand, sondern das gesamte Tetraeder der Unterrichtsebene (vgl. Prediger et al. 2017, S. 164).

Das Modell eröffnet einen erweiterten Blick auf die Professionalisierungsforschung und unterstützt die theoretische Entwicklung sowie die forschungsbasierte Optimierung entsprechender Maßnahmen (vgl. Prediger et al. 2017, S. 159f). Mit Blick auf die Qualifizierung der fortbildenden Multiplikatorinnen und Multiplikatoren kann das Tetraeder-Modell auf eine dritte Ebene transferiert werden (Abb. 2, oben). Hierbei werden die Multiplikatorinnen und Multiplikatoren selbst zu Lernenden und der Qualifizierungsgegenstand umfasst neben dem fachlichen Lerngegenstand und dem Tetraeder der Unterrichtsebene zusätzlich das Tetraeder der Fortbildungsebene (vgl. Prediger et al. 2017, S. 165).

Die der vorliegenden Studie zugrunde liegende Fortbildungsmaßnahme ist im Tetraeder-Modell des DZLM auf der Fortbildungs- und Qualifizierungsebene zu verorten, wobei die Qualifizierung der Multiplikatorinnen und Multiplikatoren hier nicht im typischen Sinne einer Schulung erfolgt. Vielmehr beobachten Multiplikatorinnen und Multiplikatoren in einem ersten Schritt eine Lehr-Lernsituation (vgl. auch Abschn. 3). Dies schafft im Sinne der Reflexion (vgl. Abschn. 2.2) ein „Spannungsfeld von Beobachtung und Selbstbeobachtung“ (de Boer 2012, S. 308), in dem sie einerseits eine konkrete Situation kritisch erkunden (im Sinne einer Fremdreflexion) und andererseits sich selbst als potenziell Agierende (im Sinne einer Selbstreflexion) bewusst in den Blick nehmen können (vgl. Bennewitz 2012). Dieses Wechselspiel zwischen Mit-Lernenden während der Beobachtung einer Fortbildungsmaßnahme und potenziellen zukünftigen Lehrenden einer ähnlichen Fortbildung kommt im Tetraeder der Fortbildungsebene auf der Seite „Lehrende - Lernende“ zum Ausdruck (vgl. Abb. 3). Dabei wird auch ein Rollenwechsel zwischen Lehrperson und Multiplikatorin bzw. Multiplikator erforderlich (vgl. Dengerink et al. 2015).

\section{Vorstellung des Gesamtrahmens der Studie und der Forschungsfrage}

Während Fortbildungen für Lehrpersonen und die Qualifizierung von Multiplikatorinnen und Multiplikatoren üblicherweise in getrennten Veranstaltungen stattfinden, 
wurden diese beiden Ebenen im Rahmen der Fortbildungsmaßnahmen der vorliegenden Studie miteinander verknüpft, indem vier Multiplikatorinnen in einem ersten Schritt eine Fortbildungsmaßnahme für fachfremd unterrichtende Sonderpädagoginnen und Sonderpädagogen hospitierten und gemeinsam reflektierten. Eine solche Reflexion kann die Reduktion des komplexen Fortbildungsgeschehens und einen fokussierteren Blick auf den Fortbildungsgegenstand mit seiner fortbildungsdidaktischen Umsetzung ermöglichen. In einem darauffolgenden Schritt wurde die Fortbildungsmaßnahme von den Multiplikatorinnen weiterentwickelt, eigenständig durchgeführt und abschließend reflektiert. Insgesamt nahmen die Multiplikatorinnen damit die in Abschn. 2.5 beschriebene Doppelrolle ein (vgl. Abb. 3).

Von besonderer Bedeutung erscheint hier die Frage, wie die Multiplikatorinnen die Teilnehmenden der Fortbildungsmaßnahme, die fachfremd unterrichtenden Sonderpädagoginnen und Sonderpädagogen, näher erfassten, da diese für die Multiplikatorinnen selbst eine eher neue Zielgruppe darstellten. Daher steht im Fokus des vorliegenden Beitrags die Forschungsfrage:

Welche Reflexionen zum Gestaltungsprinzip der Teilnehmendenorientierung können bei Multiplikatorinnen und Multiplikatoren identifiziert werden?

Nachstehend werden der konzeptionelle Rahmen sowie das methodische Vorgehen vorgestellt.

Phase 1 (05/2016-04/2017): Beobachtung einer Fortbildungsmaßnahme durch Multiplikatorinnen Die dieser Studie zugrundeliegende Fortbildungsmaßnahme zum inklusiven Mathematikunterricht für fachfremd unterrichtende Sonderpädagoginnen und Sonderpädagogen zielte darauf ab, bei den teilnehmenden Lehrpersonen ein flexibles, adaptives und fachlich sinnvolles Agieren im inklusiven Mathematikunterricht der Grundschule zu sichern. Die Themen verteilten sich auf fünf Präsenztage wie folgt (vgl. Tab. 1): ,Ziele und geeignete Lernangebote im inklusiven Mathematikunterricht“, „Zahlvorstellungen“, „Operationsvorstellungen“, „Sachrechnen“ sowie „Diagnose und Förderung“ (vgl. Scherer et al. 2019). Inhaltlich orientierte sich die Maßnahme folglich an den für das Mathematiklernen von Grundschulkindern als bedeutsam herausgestellten Basiskompetenzen (z. B. Moser Opitz 2005). Zugleich sollte die besondere Bedeutung prozessbezogener Kompetenzen (vgl. MSW 2008, S. $57 \mathrm{f}$ ) für das Lernen von Mathematik verdeutlicht werden. Bezogen auf den Fortbildungsgegenstand (vgl. Drei-Tetraeder-Modell in Abb. 2) wird dabei das Zusammenspiel fachlicher Inhalte, materialer Umsetzungen sowie möglicher Schülervorstellungen und -lösungen deutlich. Methodisch erfolgte eine Orientierung an den DZLM-Gestaltungsprinzipien (vgl. Barzel und Selter 2015; Barzel et al. 2018; vgl. Abschn. 2.1).

Die Fortbildungsmaßnahme wurde erstmalig in Nordrhein-Westfalen im Schuljahr 2016/17 mit 18 Lehrpersonen durchgeführt, evaluiert und thematisch geringfügig modifiziert (vgl. Scherer 2019; Scherer et al. 2019). Im Schuljahr 2016/17 wurde die Maßnahme mit 21 Teilnehmenden erneut durchgeführt und von vier Multiplikatorinnen begleitet. Diese wiesen als Fachleiterinnen bereits mehrjährige Erfahrung im Bereich der Erwachsenenbildung auf und waren - mit einer Ausnahme - seit 
Tab. 1 Schriftliche Beobachtungsaufträge der Multiplikatorinnen an den fünf Präsenztagen der Fortbildung

\begin{tabular}{llllll}
\hline & $\begin{array}{l}\text { 11/2016 } \\
\text { Präsenztag 1 }\end{array}$ & $\begin{array}{l}\text { 12/2016 } \\
\text { Präsenztag 2 }\end{array}$ & $\begin{array}{l}\text { 01/2017 } \\
\text { Präsenztag 3 }\end{array}$ & $\begin{array}{l}\text { 02/2017 } \\
\text { Präsenztag 4 }\end{array}$ & $\begin{array}{l}\text { 03/2017 } \\
\text { Präsenztag 5 }\end{array}$ \\
\hline $\begin{array}{l}\text { Thema } \\
\text { der Fort- } \\
\text { bildung }\end{array}$ & $\begin{array}{l}\text { Ziele und } \\
\text { geeignete } \\
\text { Lernangebote } \\
\text { im inklusiven }\end{array}$ & $\begin{array}{l}\text { Zahl- } \\
\text { vorstellungen }\end{array}$ & $\begin{array}{l}\text { Diagnose } \\
\text { und Förde- } \\
\text { rung }\end{array}$ & $\begin{array}{l}\text { Operations- } \\
\text { vorstellungen } \\
\text { \& Sach- } \\
\text { rechnen }\end{array}$ & $\begin{array}{l}\text { Halbschriftliches und } \\
\text { schriftliches Rechnen }\end{array}$ \\
& $\begin{array}{l}\text { Mathematik- } \\
\text { unterricht }\end{array}$ & & & \\
$\begin{array}{l}\text { Reflexions- } \\
\text { fokus für } \\
\text { die Mul- } \\
\text { tiplikato- } \\
\text { rinnen }\end{array}$ & $\begin{array}{l}\text { Offener } \\
\text { Beobachtungs- }\end{array}$ & $\begin{array}{l}\text { Methodische } \\
\text { auftrag }\end{array}$ & $\begin{array}{l}\text { Reflexion zur } \\
\text { Umsetzung } \\
\text { der DZLM- }\end{array}$ & $\begin{array}{l}\text { dische Reflexion zu ver- } \\
\text { schiedenen Aspekten/ } \\
\text { Reflexion zu Teilnehmen- } \\
\text { dendiskussionen }\end{array}$ & $\begin{array}{l}\text { Fachdidaktische Re- } \\
\text { flexion und inhaltliche } \\
\text { Positionierung zur Be- } \\
\text { deutung halbschrift- } \\
\text { lichen und schriftli- } \\
\text { prinzipien }\end{array}$ \\
\hline
\end{tabular}

vielen Jahren mit der Planung und Durchführung von Fortbildungen für das Fach Mathematik vertraut.

Die Multiplikatorinnen erhielten einen konkreten Vorschlag zur Strukturierung des Fortbildungsgegenstands und zur materialen Realisierung. Anhand von Beobachtungsaufträgen reflektierten sie verschiedene Aspekte schriftlich (Tab. 1; individuelle Reflexion, vgl. Abschn. 5.1), die wiederum als Basis für die kollegiale Reflexion in Phase 2 genutzt wurden (Bennewitz 2012).

\section{Phase 2 (05/2017-12/2017): Moderierte Reflexionsgespräche zum hospitierten} Fortbildungskurs durch Multiplikatorinnen Im Anschluss an die hospitierte Fortbildungsmaßnahme wurden vier moderierte Reflexionsgespräche (Gruppengespräche) mit den Multiplikatorinnen geführt, welche auf die Reflexion und Adaption der Fortbildungsmaßnahme ausgerichtet waren (Tab. 2; gemeinsame Reflexion, vgl. Abschn. 5.2). Aus der Analyse des Beobachteten sollten Chancen wie Herausforderungen des Fortbildungskonzepts diskutiert, gelungene wie misslungene Interaktionen der Teilnehmenden identifiziert und passende alternative Handlungsoptionen abgeleitet werden (vgl. Bennewitz 2012).

Phase 3 (02/2018-07/2018): Adaption, Durchführung und Evaluation des Fortbildungskurses durch Multiplikatorinnen Im Frühjahr 2018 wurde die Fortbildungsmaßnahme von den Multiplikatorinnen selbst durchgeführt, wobei ihnen die

Tab. 2 Themenfelder der vier moderierten Reflexionsgespräche mit den Multiplikatorinnen

\begin{tabular}{|c|c|c|c|c|}
\hline $\begin{array}{l}03 / 2017 \\
\text { Moderiertes } \\
\text { Reflexions- } \\
\text { gespräch } 1\end{array}$ & $\begin{array}{l}06 / 2017 \\
\text { Moderiertes } \\
\text { Reflexions- } \\
\text { gespräch } 2\end{array}$ & $\begin{array}{l}07 / 2017 \\
\text { Moderiertes } \\
\text { Reflexions- } \\
\text { gespräch } 3\end{array}$ & $09-12 / 2017$ & $\begin{array}{l}12 / 2017 \\
\text { Moderiertes } \\
\text { Reflexionsgespräch } 4\end{array}$ \\
\hline Rückschau & $\begin{array}{l}\text { Adaptions- } \\
\text { überlegungen } \\
\text { „Ergiebige Lern- } \\
\text { angebote“ }\end{array}$ & $\begin{array}{l}\text { Adaptions- } \\
\text { überlegungen } \\
\text { „Zahlvorstellun- } \\
\text { gen“ }\end{array}$ & $\begin{array}{l}\text { Planung } \\
\text { Fortbildung }\end{array}$ & $\begin{array}{l}\text { Reflexion zur durch- } \\
\text { zuführenden Fortbil- } \\
\text { dung mit Fokus auf } \\
\text { einen Baustein }\end{array}$ \\
\hline
\end{tabular}


vom DZLM entwickelten Materialien (PPTs, Arbeitsblätter, Distanzaufgaben) in adaptierbarer Form zur Verfügung standen. Die Multiplikatorinnen wurden bei der Durchführung der Fortbildungsmaßnahme ihrerseits hospitiert, und nach Abschluss der Maßnahme wurden Einzelinterviews mit den Multiplikatorinnen sowie ein abschließendes moderiertes Reflexionsgespräch mit allen Beteiligten durchgeführt und dokumentiert (vgl. auch Ratte 2019).

Der vorliegende Beitrag bezieht sich auf die ersten beiden Phasen und untersucht die Reflexionen der Multiplikatorinnen aus den individuellen schriftlichen Beobachtungen und den moderierten Reflexionsgesprächen.

\section{Methodisches Vorgehen der Datenerhebung und -auswertung}

In der vorliegenden Studie wurden die Phasen „Beobachtung - Reflexion - Adaption" miteinander verbunden, in denen die Multiplikatorinnen den Professionalisierungsgegenstand auf der einen Seite spezifizierten und strukturierten, auf der anderen Seite weiterentwickelten wie auch durchführten und auswerteten. Die Datenerhebung erfolgte auf unterschiedliche Weise:

a) Die Multiplikatorinnen ( $N=4,3$ Grundschule, 1 Förderschule), die die Maßnahme hospitierten, erhielten verschiedene Beobachtungsaufträge zur Verschriftlichung während des jeweiligen Präsenztags.

b) Es wurden moderierte Reflexionsgespräche mit den Multiplikatorinnen mit Distanz zur hospitierten Maßnahme und mit Blick auf die Adaption und Weiterentwicklung der Maßnahme durchgeführt und audiografiert.

Die individuellen schriftlichen Beobachtungen adressierten unterschiedliche Dimensionen (Tab. 1): Zunächst wurde ein offener Beobachtungsauftrag gestellt (Präsenztag 1), um an den folgenden Präsenztagen 2 bis 4 die DZLM-Gestaltungsprinzipien (vgl. Abschn. 2.1) zu fokussieren. Zu Präsenztag 5 wurde ein spezifischer mathematischer Gegenstand mit einer fachdidaktischen Positionierung in den Blick genommen. Nach der offenen Reflexion am ersten Präsenztag forderten die folgenden Aufträge eher geschlossene Reflexionen heraus (vgl. Abschn. 5.1).

Die moderierten Reflexionsgespräche (jeweils ca. $150 \mathrm{~min}$ ) nach Abschluss der Maßnahme repräsentierten eher offene Reflexionen zu unterschiedlichen Schwerpunkten (Tab. 2): Nach einer Rückschau auf die hospitierte Fortbildung standen in den Gesprächen 2 und 3 stärker Überlegungen zur Adaption im Zentrum, um nach einer Planungsphase der Multiplikatorinnen konkrete Adaptionen zu diskutieren. Während also das erste Gespräch eher retrospektive Reflexionen nahelegte, ließen die weiteren Gespräche stärker prospektive Reflexionen erwarten (vgl. Abschn. 5.2).

Den Beginn der Datenauswertung bildete die Auseinandersetzung mit den schriftlichen Reflexionen der Multiplikatorinnen. Als Grundlage für die durchgeführte inhaltlich strukturierende qualitative Inhaltsanalyse (vgl. Kuckartz 2016) dienten die vom DZLM formulierten Gestaltungsprinzipien für Fortbildungen (vgl. Barzel und Selter 2015). Das Datenmaterial wurde entsprechend der deduktiv festgelegten Hauptkategorien Kompetenzorientierung, Teilnehmendenorientierung, Lehr-Lern-Vielfalt, Fallbezug, Kooperationsanregung und Reflexionsförderung co- 
diert. Insbesondere sollte der Fragestellung nachgegangen werden, inwiefern die Teilnehmendenorientierung (vgl. Abschn. 2.1 und 2.3) in den Beobachtungen der Multiplikatorinnen eine zentrale Rolle unter den Gestaltungsprinzipien einnimmt. Anschließend wurde die Kategorie Teilnehmendenorientierung hinsichtlich eher retrospektiver und eher prospektiver Reflexionen untersucht (vgl. Abschn. 5.1).

Die Auswertung der moderierten Reflexionsgespräche orientierte sich ebenfalls am Verfahren der inhaltlich strukturierenden qualitativen Inhaltsanalyse und wurde mithilfe der Analysesoftware MAXQDA durchgeführt. Vor dem Hintergrund der Auswertung der schriftlichen Beobachtungen, welche die zentrale Bedeutung der Teilnehmendenorientierung bestätigte, wurde ein Kategoriensystem zu diesem Gestaltungsprinzip entwickelt (vgl. Tab. 5, Abschn. 5.2) und auf das in den moderierten Reflexionsgesprächen entstandene Datenmaterial angewendet. Dieses deduktiv entwickelte und induktiv angepasste Kategoriensystem umfasst neun Haupt- und sechs Subkategorien, wobei für alle Haupt- und Subkategorien inhaltliche Beschreibungen, Anwendungs- und Abgrenzungsregeln sowie Ankerbeispiele formuliert beziehungsweise ausgewählt und in einem Kategorienleitfaden festgehalten wurden. Anschließend wurde überprüft, ob es sich bei den identifizierten Codes der Hauptund Subkategorien des Gestaltungsprinzips der Teilnehmendenorientierung um eher retrospektive oder eher prospektive Reflexionen der Multiplikatorinnen handelt.

\section{Ergebnisse}

\subsection{Auswertung der schriftlichen Beobachtungsaufträge zur Teilnehmendenorientierung}

Im Rahmen einer inhaltlich strukturierenden qualitativen Inhaltsanalyse konnte der Code für die Teilnehmendenorientierung insgesamt 67 mal vergeben werden, während sich zum Vergleich etwa der Fallbezug nur in 16 Äußerungen fand (vgl. Tab. 3). Da die Fortbildung auf die Vertiefung von fachlichen und fachdidaktischen Kompetenzen abzielte, überrascht auch die häufige Nennung der Kompetenzorientierung nicht. Dass sich auch das Gestaltungsprinzip der Lehr-Lern-Vielfalt in vielen Kommentierungen der Multiplikatorinnen wiederfand, war ebenfalls zu erwarten: Kommentare zur Lehr-Lern-Vielfalt werden zwar auf einen bestimmten Inhalt der hospitierten Maßnahme bezogen, sie sind aber durchaus flexibel zu sehen und auch bei anderen Inhalten denkbar und die Multiplikatorinnen konnten hier an vielen Stellen ihr vorhandenes Wissen zu Fortbildungsdidaktik und -methodik einbringen. Die

Tab. 3 Anzahl der Codierungen zu den DZLMGestaltungsprinzipien

\begin{tabular}{ll}
\hline Gestaltungsprinzip & Anzahl der Codierungen \\
\hline Kompetenzorientierung & 58 \\
Teilnehmendenorientierung & 67 \\
Lehr-Lern-Vielfalt & 51 \\
Fallbezug & 16 \\
Kooperationsanregung & 15 \\
Reflexionsförderung & 9 \\
\hline
\end{tabular}


Dominanz der Gestaltungsprinzipien Teilnehmendenorientierung, Kompetenzorientierung und Lehr-Lern-Vielfalt deckt sich dabei mit den Ergebnissen von RoeskenWinter et al. (2015). Insgesamt war das Gestaltungsprinzip der Teilnehmendenorientierung - wie erwartet - für die Multiplikatorinnen von besonderer Wichtigkeit.

Eine differenziertere Betrachtung dieses Gestaltungsprinzips zeigte, auch wenn die schriftlichen Beobachtungsaufträge primär im Sinne einer Fremdreflexion auf die hospitierte Maßnahme ausgerichtet waren, dass sich viele Äußerungen mit Blick auf die zukünftige Maßnahme fanden, die die Multiplikatorinnen selbst durchführen würden. Dies bildete die ersten beiden Dimensionen einer retrospektiven (hinsichtlich der Hospitation) und einer eher prospektiven Reflexion (hinsichtlich der eigenen Durchführung) ab (vgl. Abschn. 2.2).

Die retrospektiven Reflexionen haben hierbei eher normativen Charakter, was durch spezifische Reflexionsaufträge auch nahegelegt wird. Diese normativen Reflexionen beziehen sich einerseits auf die konkrete Fortbildung und bewerten unter Umständen spezifische beobachtete Aspekte. Sie können aber auch weniger konkret formuliert sein und damit eine eher allgemeine Positionierung repräsentieren. Ebenso wie die normative Dimension kann auch die prospektive Dimension eher allgemeine Überlegungen repräsentieren oder aber bereits Hinweise auf die Adaptionen geben, die die Multiplikatorinnen tatsächlich vornehmen werden.

Theoretisch wurden zunächst die von Sherin und Drake (2009) herausgestellten prospektiven Adaptionsprozesse (vgl. Abschn. 2.2) aufgegriffen und ausdifferenziert.

Allerdings zeigte sich im Codierungsprozess, dass „kreative Neugestaltungen“ (create) nicht trennscharf von spezifischen Erweiterungen beziehungsweise additiven Elementen zu unterscheiden waren. So wurde unter anderem zum Aufgabenformat „Rechendreieck“ (Thema Ergiebige Lernangebote) der Vorschlag geäußert, bei der Umsetzung in der Fortbildung noch „,Wortspeicher/Satzanfänge/Forschermittel“ zu integrieren. Dieser Vorschlag bedeutet einerseits, neue Elemente mit dem Fokus auf Sprache hinzuzunehmen, andererseits erweitert und ergänzt er die bereits bestehenden Ausführungen zur Behandlung des Aufgabenformats „Rechendreieck“. Daher wurde der Adaptionsprozess unter der Dimension erweiternd/additiv \& kreativ/erneuernd zusammengefasst.

Analog dazu wurde der Prozess des Reduzierens als reduzierend/subtraktive Dimension und der Prozess des Ersetzens als alternativ/ersetzende Dimension herausgearbeitet. In Abgrenzung zu Leufer et al. (2019), die zu den zentralen Adaptionsprozessen von Sherin und Drake (2009) weitere Ausdifferenzierungen identifizieren (beispielsweise das Aufteilen einer thematischen Sitzung auf mehrere Sitzungen oder das Verändern einer thematischen Reihenfolge), konnten bei der Auswertung der schriftlichen Beobachtungsaufträge keine derartig detaillierten Adaptionen festgestellt werden. Dies ist vermutlich darauf zurückzuführen, dass sich die vier Multiplikatorinnen noch nicht im konkreten Adaptionsprozess befanden.

Bei den prospektiven Reflexionen wurden am häufigsten erweiternd/additiv \& kreativ/erneuernde Aspekte benannt (66 Codes), während der Code für alternativ/ ersetzende Aspekte nur 16 mal und der Code für reduzierend/subtraktive Aspekte lediglich 4 mal vergeben wurde. 
Tab. 4 Normative und prospektive Reflexionen mit Ankerbeispielen

\begin{tabular}{|c|c|c|}
\hline & Ankerbeispiel & Perspektive \\
\hline \multicolumn{3}{|c|}{$\begin{array}{l}\text { Normative Reflexion } \\
\text { Dimension }\end{array}$} \\
\hline $\begin{array}{l}\text { Bestätigend/ } \\
\text { positiv }\end{array}$ & $\begin{array}{l}\text { „Die Erfahrungen bei den Hausaufgaben wurden gut aufgegriffen } \\
\text { und gut kommentiert. “ (Präsenztag 3: Diagnose \& Förderung) }\end{array}$ & $\begin{array}{l}\text { Hospitierte } \\
\text { Fortbildung }\end{array}$ \\
\hline $\begin{array}{l}\text { Herausfordernd/ } \\
\text { kritisch }\end{array}$ & $\begin{array}{l}\text { „Nicht alle Teilnehmenden wissen um die Verflechtung der in- } \\
\text { halts- und prozessbezogenen Kompetenzen im Fach Mathematik } \\
\text { bzw. um deren Chancen." } \\
\text { (Präsenztag 1: Ziele und geeignete Lernangebote im inklusiven } \\
\text { Mathematikunterricht) }\end{array}$ & $\begin{array}{l}\text { Spezifische } \\
\text { Teilnehmen- } \\
\text { dengruppe }\end{array}$ \\
\hline \multicolumn{3}{|c|}{$\begin{array}{l}\text { Prospektive Reflexion } \\
\text { Dimension }\end{array}$} \\
\hline $\begin{array}{l}\text { Erweiternd/ } \\
\text { additiv \& } \\
\text { kreativ/ } \\
\text { erneuernd }\end{array}$ & $\begin{array}{l}\text { „Frage [der Teilnehmenden] nach der Vielfalt der Rechenwege } \\
\text { - für alle Kinder, alle Wege? - verlangt wieder eine Positionie- } \\
\text { rung der Referentin.“ } \\
\text { (Präsenztag 4: Operationsvorstellungen \& Sachrechnen) }\end{array}$ & $\begin{array}{l}\text { Hospitierte } \\
\text { \& zukünftige } \\
\text { Fortbildung }\end{array}$ \\
\hline $\begin{array}{l}\text { Reduzierend/ } \\
\text { subtraktiv }\end{array}$ & $\begin{array}{l}\text { [Operatoraspekt, Rechenzahlaspekt; Anm. Autoren] „war auch in } \\
\text { dem Bild nicht relevant - besser rauslassen?“ } \\
\text { (Präsenztag 2: Zahlvorstellungen) }\end{array}$ & $\begin{array}{l}\text { Hospitierte } \\
\& \text { zukünftige } \\
\text { Fortbildung }\end{array}$ \\
\hline $\begin{array}{l}\text { Alternativ/ } \\
\text { ersetzend }\end{array}$ & $\begin{array}{l}\text { „Alternativ: Die Sonderpädagogen mit dem Förderschwerpunkt } \\
\text { Sprache zu Wort kommen lassen. “ (Alternative zur Thematisie- } \\
\text { rung sprachlicher Anforderungen in Testinstrumenten) } \\
\text { (Präsenztag 3: Diagnose \& Förderung) }\end{array}$ & $\begin{array}{l}\text { Zukünftige } \\
\text { Fortbildung }\end{array}$ \\
\hline
\end{tabular}

Insgesamt fanden sich mitunter direkte Beziehungen zwischen normativer und prospektiver Reflexion. So wurde zum Beispiel zu einem herausfordernden Aspekt (Bsp. ,Ich hatte den Eindruck, dass das [Prozessbezogene Kompetenzen; Anm. Autoren] für einige [Teilnehmende; Anm. Autoren] doch sehr abstrakt war.") direkt eine mögliche Adaption geäußert: „Inhaltlich bin ich der Meinung, dass man unbedingt die prozessbezogenen Kompetenzen illustrieren müsste, vielleicht anhand eines der Aufgabenbeispiele und der damit verbundenen Fragestellungen. So könnte auch deutlich werden, dass je nach gewählter Frage für die Kinder der eine oder andere Bereich stärker fokussiert und ein Schwerpunkt gesetzt werden kann. “ (Präsenztag 1: Ziele und geeignete Lernangebote im inklusiven Mathematikunterricht).

Gleichwohl wurden nicht zwangsläufig zu normativ kritisch reflektierten Aspekten auch prospektiv additive oder ersetzende Alternativen formuliert. Manche herausfordernden Aspekte blieben in den schriftlichen Reflexionen ohne prospektive Reflexionen für die eigene zukünftige Maßnahme.

Die konkreten Äußerungen zur Teilnehmendenorientierung in den verschiedenen Dimensionen bezogen sich auf Inhalte oder methodische Aspekte, auf strukturelle Überlegungen sowie auf das generelle Setting. In Tab. 4 werden die Dimensionen differenziert mit jeweils einem Ankerbeispiel (mit Angabe des jeweiligen Themas; vgl. Abschn. 3 bzw. Tab. 1 in Abschn. 4) und der primären Ausrichtung (Perspektive) genauer vorgestellt. 


\subsection{Auswertung der moderierten Reflexionsgespräche zur Teilnehmendenorientierung}

Nachdem sich die zentrale Bedeutung des Gestaltungsprinzips der Teilnehmendenorientierung bei der Analyse der schriftlichen Beobachtungen bestätigte, wurde dieses Gestaltungsprinzip in den deutlich umfangreicheren moderierten Reflexionsgesprächen noch einmal differenzierter betrachtet. Dazu wurde auf der Grundlage der einschlägigen Literatur zum Bereich der Lehrerfortbildung und zum Gestaltungsprinzip der Teilnehmendenorientierung (vgl. u.a. Abschn. 2.3 und 2.4) ein deduktives Kategoriensystem herausgearbeitet, das im ersten Auswertungsschritt genutzt und während der Analyse induktiv erweitert wurde. Im Anschluss konnten die identifizierten Reflexionen der Multiplikatorinnen zum Gestaltungsprinzip der Teilnehmendenorientierung - ähnlich wie bei der Analyse der schriftlichen Beobachtungen - hinsichtlich der ihnen zugrundeliegenden normativen oder prospektiven Dimensionen untersucht werden. Das deduktiv entwickelte Kategoriensystem umfasste die nachstehenden neun Hauptkategorien sowie sechs Subkategorien (Tab. 5):

Die Subkategorie Sonderpädagogisches Wissen der Teilnehmenden (KV-SW-T) setzt sich insofern von den anderen Subkategorien ab, als dass hier Reflexionen über das Wissen der Sonderpädagoginnen und Sonderpädagogen einzuordnen wären, das Teil ihrer universitären Ausbildung ist und damit in der Regel über das Professionswissen von Regelschullehrpersonen hinausreichen dürfte. Äußerungen über die Diagnostische Kompetenz der Teilnehmenden (KV-DK-T) wurden im Rah-

Tab. 5 Anzahl der Codierungen deduktiv entwickelter Haupt- und Subkategorien

\begin{tabular}{lll}
\hline Deduktiv entwickelte Haupt- und Subkategorien & Code & $\begin{array}{l}\text { Anzahl der } \\
\text { Codierungen }\end{array}$ \\
\hline $\begin{array}{l}\text { Persönlichkeitsbezogene und berufsbiographische Voraussetzungen der } \\
\text { Teilnehmenden }\end{array}$ & $P B V-T$ & 9 \\
Kognitive Voraussetzungen der Teilnehmenden & $K V-T$ & 81 \\
Sonderpädagogisches Wissen der Teilnehmenden & $K V-$ & 6 \\
& $S W-T$ & \\
(Allgemein-)Pädagogisches Wissen der Teilnehmenden & $K V-$ & 4 \\
& $P W-T$ & \\
Fachliches und fachdidaktisches Wissen der Teilnehmenden & $K V-$ & 53 \\
& $F F W-T$ & \\
Diagnostische Kompetenz der Teilnehmenden & $K V-$ & 6 \\
& $D K-T$ & \\
Organisations- und Beratungswissen der Teilnehmenden & $K V-$ & 1 \\
& $O B W-T$ & \\
Sonstige kognitive Voraussetzungen & $K V-S-T$ & 11 \\
Überzeugungen und Werthaltungen der Teilnehmenden & $\ddot{U W-T}$ & 15 \\
Selbstregulative Fähigkeiten der Teilnehmenden & $S F-T$ & 10 \\
Motivationale Orientierungen der Teilnehmenden & $M O-T$ & 4 \\
Bedarfe und Interessen der Teilnehmenden & $B I-T$ & 24 \\
Akzeptanz der Fortbildungsmaßnahme auf Seiten der Teilnehmenden & $A F-T$ & 20 \\
Schulkontext der Teilnehmenden & $S K-T$ & 137 \\
Sonstiges Teilnehmendenorientierung & $S-T$ & 11 \\
\hline
\end{tabular}


men des vorliegenden Projekts gesondert erfasst und nicht unter die Subkategorie $K V$-SW-T subsummiert. So stellt die diagnostische Kompetenz sowohl einen zentralen Bestandteil der professionellen Handlungskompetenz von Sonderpädagoginnen und Sonderpädagogen (vgl. u. a. Moser und Kropp 2015) als auch von Regelschullehrpersonen dar (vgl. u. a. Baumert und Kunter 2006).

Das deduktiv entwickelte Kategoriensystem wurde während eines ersten Analysedurchgangs weiter ausdifferenziert, da die Multiplikatorinnen nicht nur die kognitiven Voraussetzungen der Teilnehmenden vor Antritt der Fortbildungsmaßnahme betrachteten, sondern auch die Weiterentwicklung ihrer kognitiven Voraussetzungen beziehungsweise die Entwicklung neuer Wissensbestände und Kompetenzen. Diese Beobachtung wurde in das Kategoriensystem integriert, indem alle Subkategorien der Hauptkategorie Kognitive Voraussetzungen der Teilnehmenden (KV-T) sowie die Hauptkategorie Überzeugungen und Werthaltungen der Teilnehmenden ( $\ddot{U} W$-T) nochmals in vor der Fortbildung (KV-T-Prä beziehungsweise $\ddot{U} W$-T-Prä) und nach der Fortbildung ( $K V$-T-Post beziehungsweise $\ddot{U} W$-T-Post) unterteilt wurden. Darüber hinaus fiel auf, dass die Multiplikatorinnen an mehreren Stellen über wahrgenommene Ängste und Sorgen der Teilnehmenden sprachen, weshalb das Kategoriensystem um die induktiv gebildete Hauptkategorie Ängste und Sorgen der Teilnehmenden ( ̈̈S-T) erweitert wurde. Ferner wurde deutlich, dass sich die Multiplikatorinnen intensiv darüber austauschten, wie den Teilnehmenden der Zugang zu bestimmten Fortbildungsinhalten erleichtert werden kann. Auch wenn die entsprechenden Reflexionen auf die Teilnehmenden ausgerichtet waren, handelte es sich hierbei vorwiegend um fortbildungsdidaktische und -methodische Überlegungen, welche im Rahmen der vorliegenden Publikation nicht im Mittelpunkt stehen. Kommentare der Multiplikatorinnen, in welchen sie sich in besonderem Maße wertschätzend gegenüber den Teilnehmenden äußerten, wurden unter der induktiv gebildeten Hauptkategorie Wertschätzung der Teilnehmenden durch die Multiplikatorinnen $(W-T)$ zusammengefasst. In einem zweiten Schritt wurden die identifizierten Codes aller Kategorien zum Gestaltungsprinzip der Teilnehmendenorientierung den verschiedenen normativen und prospektiven Reflexionsebenen zugeordnet ${ }^{4}$.

Die Hauptkategorie Schulkontext der Teilnehmenden (SK-T) konnte mit Abstand am häufigsten codiert werden (137 Codes), wobei alle Reflexionen der Multiplikatorinnen zusammengetragen wurden, die sich auf den schulischen Alltag der Sonderpädagoginnen und Sonderpädagogen bezogen. Das kann ihre Rollen und Funktionen innerhalb des Klassenkontexts oder des Lehrerkollegiums wie auch die ihnen zugetragene Unterstützung seitens des Kollegiums oder der Schulleitung umfassen (vgl. Lipowsky und Rzejak 2017). Zudem wurden Äußerungen der Multiplikatorinnen berücksichtigt, welche die Schulformen und -stufen der Sonderpädagoginnen und Sonderpädagogen betrafen oder formale Aspekte der Fortbildungsgestaltung, wie

\footnotetext{
4 Da sich einige Äußerungen der Multiplikatorinnen zu mehreren Kategorien des Gestaltungsprinzips der Teilnehmendenorientierung zuordnen ließen, ist es an diesen Stellen - auch im Hinblick auf die Zuordnung zu den verschiedenen Reflexionsdimensionen - zu einer Mehrfachcodierung von Aussagen gekommen. Darüber hinaus wiesen einzelne Äußerungen gleichzeitig sowohl eine normative als auch eine prospektive Reflexionsdimension auf, wodurch es zur Doppelcodierung entsprechender Äußerungen kam.
} 
Tab. 6 Schulkontext der Teilnehmenden $(S K-T)$ - Normative und prospektive Reflexionen mit Ankerbeispielen

\begin{tabular}{|c|c|c|c|}
\hline Kategorie & Ankerbeispiel & Reflexion & Perspektive \\
\hline SK-T & $\begin{array}{l}\text { „Und das Andere was mir aufgefallen ist, ähm, die } \\
\text { kommen aus schulischen Kontexten, wo diese Auf- } \\
\text { gaben selber nicht etabliert sind. Der Unterricht ist } \\
\text { ein anderer als der, der jetzt hier so vorgestellt wur- } \\
\text { de. Überwiegend jedenfalls.“ (Reflexionsgespräch } 1 \text {, } \\
\text { Z. 24) }\end{array}$ & $\begin{array}{l}\text { Normativ her- } \\
\text { ausfordernd/ } \\
\text { kritisch }\end{array}$ & $\begin{array}{l}\text { Spezifische } \\
\text { Teilnehmenden- } \\
\text { gruppe und } \\
\text { hospitierte } \\
\text { Fortbildung }\end{array}$ \\
\hline SK-T & $\begin{array}{l}\text { „Wenn ich das Prinzip von Entdeckerpäckchen ver- } \\
\text { standen habe, kann man Teilnehmer ja auch bitten: So, } \\
\text { und was könnten Ihre Kinder da leisten? Ne? Das, und } \\
\text { dann so die Erfahrung zu machen: Ah, Moment. Das } \\
\text { kann ich ja runterbrechen nochmal.“ (Reflexionsge- } \\
\text { spräch } 1, \text { Z. } 488 \text { ) }\end{array}$ & $\begin{array}{l}\text { Prospektiv } \\
\text { erweiternd/ } \\
\text { additiv \& } \\
\text { kreativ/ } \\
\text { erneuernd }\end{array}$ & $\begin{array}{l}\text { Zukünftige } \\
\text { Fortbildung }\end{array}$ \\
\hline
\end{tabular}

etwa die Anzahl und den zeitlichen Abstand der Präsenztermine sowie die Freistellungsmöglichkeiten seitens der Schulen.

Die zahlreichen Reflexionen der Multiplikatorinnen, in denen sich der Blick auf die vorwiegend sonderpädagogische Schülerschaft der Teilnehmenden richtete, wurden ebenfalls unter der Kategorie $S K-T$ zusammengefasst (vgl. Tab. 6).

Die Reflexionen der Multiplikatorinnen konnten auch in der Hauptkategorie Schulkontext der Teilnehmenden (SK-T) einer eher normativen (ca. 80\%) oder einer eher prospektiven (ca. 20\%) Dimension zugeordnet werden (vgl. Tab. 6). Von den normativen Reflexionen bezogen sich ca. $70 \%$ auf die spezifische Teilnehmendengruppe und ca. 30\% auf die hospitierte Fortbildungsmaßnahme. Der Großteil der normativen Reflexionen, die sich auf die spezifische Teilnehmendengruppe bezogen (ca. 75\%), wurden als herausfordernd/kritisch eingestuft. Eine Verknüpfung dieser herausfordernd/kritischen Reflexionen mit einer prospektiven Reflexionsdimension erfolgte in nur knapp $10 \%$ der Fälle.

In Bezug auf die hospitierte Fortbildungsmaßnahme konnten ca. 35\% der normativen Reflexionen als bestätigend/positiv kodiert werden, während weitere ca. $60 \%$ eher herausfordernd/kritisch waren. Ca. 25\% dieser normativ herausfordernd/ kritischen Reflexionen in Bezug auf die hospitierte Fortbildung wurden ${ }^{5}$, direkt mit einer prospektiven Reflexionsdimension - also beispielsweise mit einer Idee zur alternativen Gestaltung der Fortbildung - verknüpft. „Das ist vielleicht auch genau die Ebene M4, die du gerade angesprochen hast, die man stärker fokussieren müsste. Immer noch oder immer wieder diesen, diesen Bezug zur eigenen Situation herstellen. Das finde ich, das kommt auch zu kurz. Also Info und dann: Was bedeutet das für uns?" (Reflexionsgespräch 3, Z. 49).

\footnotetext{
5 Knapp 5\% der normativen Äußerungen der Multiplikatorinnen, welche der Hauptkategorie SK-T zugeordnet waren, konnten keiner der beiden Reflexionsebenen herausfordernd/kritisch oder bestätigend/ positiv zugeordnet werden. Sie wurden folglich als ,neutrale“ normative Reflexionen eingestuft. Insgesamt traf dieser Fall auf knapp 10\% aller normativen Reflexionen zu, die sich auf das Gestaltungsprinzip der Teilnehmendenorientierung beziehen.
} 
Betrachtet man wieder die übergeordnete Ebene des Gestaltungsprinzips der Teilnehmendenorientierung, wurden in der Hauptkategorie Kognitive Voraussetzungen der Teilnehmenden $(K V-T)$ die Einschätzungen der Multiplikatorinnen hinsichtlich der vorhandenen oder nicht vorhandenen kognitiven Voraussetzungen der Teilnehmenden zusammengetragen. Dabei wurden sowohl theoretisch-formale als auch praktische Wissensbestände (vgl. Baumert und Kunter 2006, S. 483) beziehungsweise praktische Erfahrungen der Teilnehmenden berücksichtigt. Obwohl dem Professionswissen der Sonderpädagoginnen und Sonderpädagogen eine besondere Bedeutung zukommt, fallen auch allgemeine kognitive Voraussetzungen der Teilnehmenden in diese Hauptkategorie. Insgesamt wurde die Hauptkategorie $K V-T 81$ mal codiert (vgl. Tab. 5). Der Großteil der Codierungen war dabei der Subkategorie Fachliches und fachdidaktisches Wissen der Teilnehmenden-Prä (KV-FFW-Prä) (43 Codes) zuzuordnen, welche Reflexionen bezüglich des vorhandenen oder nicht vorhandenen fachlichen und fachdidaktischen Wissens einschließt. In Summe bezogen sich hier fast doppelt so viele Reflexionen auf das nicht vorhandene beziehungsweise das noch zu erwerbende fachliche und fachdidaktische Wissen der Teilnehmenden als auf ihr bereits vorhandenes fachliches und fachdidaktisches Wissen. Dies lässt vermuten, dass die Fachfremde der teilnehmenden Sonderpädagoginnen und Sonderpädagogen eine besondere Rolle für die Multiplikatorinnen spielte.

Die Wissensbereiche des fachlichen und fachdidaktischen Wissens wurden hier zu einer gemeinsamen Kategorie zusammengefasst, da verschiedene qualitative Untersuchungen (vgl. u. a. Leinhardt et al. 1991; Ma 1999) zeigen konnten, „dass das tatsächlich im Unterricht verfügbare fachdidaktische Handlungsrepertoire von Lehrkräften weitgehend von der Breite und Tiefe ihres konzeptuellen Fachverständnisses abhängt" (Baumert und Kunter 2006, S. 492).

Auch hinsichtlich der Hauptkategorie Kognitive Voraussetzungen der Teilnehmenden $(K V-T)$ war eine Zuordnung der einzelnen Äußerungen zu den verschiedenen normativen und prospektiven Reflexionsdimensionen möglich (vgl. Tab. 7 für KVFFW-T Prä). Ähnlich wie in der Hauptkategorie Schulkontext der Teilnehmenden $(S K-T)$ bezog sich der überwiegende Anteil der normativen Reflexionen auf die spe-

Tab. 7 Kognitive Voraussetzungen der Teilnehmenden-Prä (KV-FFW-T-Prä) - Normative und prospektive Reflexionen mit Ankerbeispielen

\begin{tabular}{|c|c|c|c|}
\hline Kategorie & Ankerbeispiel & Reflexion & Perspektive \\
\hline $\begin{array}{l}\text { KV- } \\
\text { FFW-T } \\
\text { Prä }\end{array}$ & $\begin{array}{l}\text { „Und ob man in diesen mathematischen Bereich } \\
\text { dann vielleicht für einige, die es möchten, noch- } \\
\text { mal ergänzend anbietet, weil einige, hatte ich auch } \\
\text { den Eindruck, die brauchten das nicht. Die waren } \\
\text { fachlich, fand ich, ziemlich fit, mathematisch." } \\
\text { (Reflexionsgespräch } 1, \mathrm{Z} .114)\end{array}$ & $\begin{array}{l}\text { Normativ } \\
\text { bestätigend/positiv } \\
\text { Prospektiv erwei- } \\
\text { ternd/additiv \& } \\
\text { kreativ/erneuernd }\end{array}$ & $\begin{array}{l}\text { Spezifische } \\
\text { Teilnehmen- } \\
\text { dengruppe } \\
\text { und zukünf- } \\
\text { tige Fortbil- } \\
\text { dung }\end{array}$ \\
\hline $\begin{array}{l}\text { KV- } \\
\text { FFW-T } \\
\text { Prä }\end{array}$ & $\begin{array}{l}\text { „Ich finde, es ist auch nicht nur mit der Sach- } \\
\text { analyse getan und mit, mit ergiebigen Aufgaben, } \\
\text { sondern es gibt auch eine bestimmte Didaktik, die } \\
\text { klug und sinnvoll ist. (.) Und das müssen Sonder- } \\
\text { pädagogen wissen. Was kommt zuerst? Was mache } \\
\text { ich dann? Wie baue ich das auf? Und das wissen } \\
\text { sie häufig nicht.“ } \\
\text { (Reflexionsgespräch 2, Z. 1085) }\end{array}$ & $\begin{array}{l}\text { Normativ } \\
\text { herausfordernd/ } \\
\text { kritisch }\end{array}$ & $\begin{array}{l}\text { Spezifische } \\
\text { Teilnehmen- } \\
\text { dengruppe }\end{array}$ \\
\hline
\end{tabular}


Tab. 8 Bedarfe und Interessen der Teilnehmenden (BI-T) - Normative Reflexion mit Ankerbeispiel

\begin{tabular}{llll}
\hline Kategorie & Ankerbeispiel & Reflexion & Perspektive \\
\hline BI-T & „Wobei ich finde schon, dass die auch mit diesem Prob- & Normativ & Spezifische \\
& lem kommen. Ich finde, wir haben das ganz deutlich ge- & herausfordernd/ & Teilnehmen- \\
& spürt in allen Sitzungen, dass die immer wieder auch, & kritisch & dengruppe \\
& äh, dieses Problem angebracht haben: Ich habe ja nun & & und hospitier- \\
& mal diese Kinder und ich kann das so mit meinen Kin- & & te Fortbildung \\
& dern nicht machen. Also das finde ich, kam immer wieder & & \\
& raus.“ & & \\
& & \\
\end{tabular}

zifische Teilnehmendengruppe. Hinsichtlich der Subkategorie Fachliches und fachdidaktisches Wissen der Teilnehmenden vor der Fortbildung (KV-FFW-T Prä) waren zum Beispiel $80 \%$ der normativen Reflexionen auf die Teilnehmendengruppe und nicht auf die Fortbildung als solche ausgerichtet. So haben es die Multiplikatorinnen beispielsweise als durchaus herausfordernd empfunden, dass Fachbegriffe von den Teilnehmenden oftmals nicht gekannt oder nicht korrekt verwendet wurden. „Und dieser Begriff wird dann auch bitte verwendet. Und nicht irgendwie umschrieben. So. Ne? Und, und, und da sehe ich die Bedarfe. In dieser fachlichen Genauigkeit." (Reflexionsgespräch 2, Z. 1093). Auch in der Hauptkategorie Kognitive Voraussetzung der Teilnehmenden $(K V-T)$ schlossen sich in vergleichsweise wenigen Fällen direkte prospektive Reflexionen an.

Insgesamt konnten alle weiteren Haupt- und Subkategorien des Gestaltungsprinzips der Teilnehmendenorientierung deutlich seltener codiert werden als die Hauptkategorien Schulkontext der Teilnehmenden (SK-T) und Kognitive Voraussetzungen der Teilnehmenden ( $K V-T)$ (vgl. Tab. 5). An dritter Stelle kann die Hauptkategorie Bedarfe und Interessen der Teilnehmenden (BI-T) (24 Codes) benannt werden, die Reflexionen der Multiplikatorinnen umfasst, die sich auf die Bedarfe, Interessen, Wünsche oder auch Probleme der Teilnehmenden bezogen (vgl. Tab. 8). Dabei kann es sich sowohl um die Wiedergabe von Aussagen handeln, welche die Teilnehmenden während der hospitierten Fortbildungsmaßnahme getätigt haben, als auch um schlichte Vermutungen der Multiplikatorinnen, die zum Beispiel die Beweggründe der Sonderpädagoginnen und Sonderpädagogen, an der spezifischen Fortbildungsmaßnahme teilzunehmen, betrafen. Grundsätzlich war auch hier eine Differenzierung zwischen normativen (ca. 70\%) und prospektiven Reflexionen (ca. 30\%) möglich.

Ähnlich häufig wie die Hauptkategorie BI-T wurde die Hauptkategorie Akzeptanz der Fortbildungsmaßnahme auf Seiten der Teilnehmenden (AF-T) (20 Codes) codiert (vgl. Tab. 5). Diese Kategorie umfasst alle Reflexionen, die Aufschluss über die Akzeptanz der hospitierten Fortbildungsmaßnahme auf Seiten der Teilnehmenden gaben (vgl. Tab. 9). Hierbei kann es sich um Äußerungen der Multiplikatorinnen handeln, die sich auf - von den Teilnehmenden selbst geäußerte - positive oder negative Bewertung der Fortbildungsmaßnahme, -ziele oder einzelner Fortbildungsaktivitäten bezogen. Zudem wurden Reflexionen codiert, welche Bezug auf die von den Multiplikatorinnen wahrgenommene Bereitschaft beziehungsweise Motivation der Teilnehmenden nahmen, sich aktiv in die Fortbildung einzubringen, oder aufgekommene Widerstände beleuchteten. In dieser Kategorie ließen sich lediglich zwei prospektive Reflexionen ausfindig machen. Darüber hinaus konnten etwa doppelt so 
Tab. 9 Akzeptanz der Fortbildungsmaßnahme auf Seiten der Teilnehmenden (AF-T) - Normative Reflexion mit Ankerbeispiel

\begin{tabular}{llll}
\hline Kategorie & Ankerbeispiel & Reflexion & Perspektive \\
\hline AF-T & $\begin{array}{l}\text { „Ja. Das wollen wir jetzt nicht. Ich hab' da Schüler, die, } \\
\text { die können nicht sehen und die. So. Und ich soll jetzt } \\
\text { 'n Plakat zum Minusturm machen, also da war so, ja, } \\
\begin{array}{l}\text { Unmut einfach.“ } \\
\text { (Reflexionsgespräch 1, Z. 50) }\end{array}\end{array}$ & $\begin{array}{l}\text { Normativ } \\
\text { herausfordernd/ } \\
\text { kritisch }\end{array}$ & Fospitierte \\
Fortbildung \\
\end{tabular}

viele normativ herausfordernd/kritische wie normativ bestätigend/positive Reflexionen gezählt werden.

Insgesamt ließen sich nur wenige Reflexionen der Multiplikatorinnen im Hinblick auf das sonderpädagogische und (allgemein-)pädagogische Wissen der Teilnehmenden sowie ihre diagnostische Kompetenz finden (vgl. Tab. 5). Während die Subkategorie Sonderpädagogisches Wissen der Teilnehmenden vor der Fortbildung $(K V$-SW-T-Prä) noch immerhin sechsmal codiert werden konnte, fanden sich lediglich vier Aussagen, die sich auf die Diagnostische Kompetenz der Teilnehmenden vor der Fortbildung ( $K V$-DK-T-Prä) bezogen und nur zwei Äußerungen, welche die Diagnostische Kompetenz der Teilnehmenden nach der Fortbildung (KV-DK-T-Post) in den Blick nahmen. Dieses Ergebnis erscheint insofern unerwartet, als dass das Thema „Diagnose“ expliziter Fortbildungsgegenstand der Veranstaltung war. Das (Allgemein-)Pädagogische Wissen der Teilnehmenden vor wie nach der Fortbildung $(K V$ - $P W$-T-Prä beziehungsweise $K V$ - $P W$-T-Post) scheint eine noch geringere Rolle für die Multiplikatorinnen gespielt zu haben. Es fanden sich jeweils nur zwei Codierungen. Das Organisations- und Beratungswissen der Teilnehmenden (KV-OBW-T) wurde von den Multiplikatorinnen an nur einer Stelle in die Diskussion eingebracht.

Abschließend sei angemerkt, dass sich in den moderierten Reflexionsgesprächen insgesamt deutlich mehr normative (ca. 80\%) als prospektive (ca. 20\%) Reflexionen der Multiplikatorinnen finden ließen. Eine Überschneidung normativer und prospektiver Reflexionen wurde in lediglich $10 \%$ der Fälle festgestellt. In Bezug auf die normativen Reflexionen wurden deutlich mehr herausfordernd/kritische $(65 \%)$ als bestätigend/positive (25\%) Reflexionen codiert. Weitere $10 \%$ wurden als ,neutral“ eingestuft. Bei den prospektiven Reflexionen überwogen - noch deutlicher als in den schriftlichen Beobachtungsaufträgen - jene, die als erweiternd/additiv \& kreativ/erneuernd eingeschätzt wurden (ca. 80\%). Wird die Gesamtheit aller gezählten prospektiven Reflexionen - unabhängig ihrer Reflexionsdimension - in den Blick genommen, so schlossen sich rund 35\% dieser Reflexionen an Äußerungen der Multiplikatorinnen an, die als herausfordernd/kritisch eingeordnet wurden. Knapp $10 \%$ wurden im Zusammenhang mit bestätigend/positiven normativen Reflexionen geäußert. Weitere $40 \%$ standen für sich allein und wiesen keinen direkten Bezug zu einer normativen Reflexionsdimension auf. 


\section{Diskussion und Ausblick}

Multiplikatorinnen und Multiplikatoren stehen immer wieder vor der Herausforderung, ein fachdidaktisch relevantes Thema für nicht im Fach ausgebildete Lehrpersonen so aufzubereiten, dass diese zukünftig fachdidaktisch gehaltvollen Unterricht planen, durchführen und reflektieren können. Wie sie dieser Herausforderung begegnen oder bei deren Bewältigung unterstützt werden können, ist bislang jedoch wenig erforscht. Folglich ist es bedeutsam, empirisch gesicherte Erkenntnisse über das berufliche Handeln von Multiplikatorinnen und Multiplikatoren im Hinblick auf die Planung und Durchführung fachbezogener Fortbildungsmaßnahmen zu generieren und damit Grundlagen für ihre Professionalisierung zu schaffen.

An Überlegungen zur teilnehmenden Unterrichtsbeobachtung (de Boer 2012) und die Idee des erfahrungsbasierten reflexiven Lernens (Dewey 2000, 2002) anknüpfend war es das Ziel des Beitrags, die Reflexionen von Multiplikatorinnen und Multiplikatoren bei der Beobachtung und anschließenden Planung einer Fortbildungsmaßnahme für fachfremd unterrichtende Sonderpädagoginnen und Sonderpädagogen zu untersuchen. Hierzu hospitierten vier Multiplikatorinnen bei der Durchführung einer vom DZLM entwickelten Fortbildungsmaßnahme zum inklusiven Mathematikunterricht mit der Perspektive, diese Maßnahme zukünftig selbstständig durchzuführen. Die Multiplikatorinnen verschriftlichten ihre Beobachtungen während der Hospitation der Fortbildung und erhielten nach Abschluss der Maßnahme die Gelegenheit, sich in moderierten Reflexionsgesprächen über das Beobachtete auszutauschen. Empirische Erkenntnisse berücksichtigend, wonach die Orientierung an den (Vor-)Kenntnissen und Bedarfen der Teilnehmenden für den Erfolg von Fortbildungsmaßnahmen entscheidend ist (Barzel und Selter 2015; Lipowsky und Rzejak 2017), wurde der Forschungsfrage nachgegangen, welche Reflexionen zum Gestaltungsprinzip der Teilnehmendenorientierung die Multiplikatorinnen zeigten.

Die Auswertung der individuellen schriftlichen Reflexionen machte deutlich, dass die Multiplikatorinnen dem Gestaltungsprinzip der Teilnehmendenorientierung tatsächlich eine besondere Aufmerksamkeit schenkten. So konnte der Code für die Teilnehmendenorientierung deutlich häufiger vergeben werden als die Codes für die DZLM-Gestaltungsprinzipien Fallbezug, Reflexionsförderung oder Kooperationsanregung (vgl. auch die Ergebnisse von Roesken-Winter et al. 2015). Dies liegt womöglich daran, dass die Multiplikatorinnen einerseits aufgrund ihrer bisherigen Fortbildungserfahrungen um die zentrale Bedeutung des Gestaltungsprinzips der Teilnehmendenorientierung wissen und andererseits auf Grund ihrer geringeren Erfahrungen mit der Gruppe der fachfremd unterrichtenden Sonderpädagoginnen und Sonderpädagogen diese Teilnehmenden besonders beachten. Daneben wäre zu erwarten gewesen, dass die Multiplikatorinnen auch das Gestaltungsprinzip der Kooperationsanregung öfter in den Blick nehmen, da die Kooperation in multiprofessionellen Teams nach wie vor eine Herausforderung für den inklusiven Unterricht darstellt (Heinrich und Werning 2013). Dass dieser Code weniger häufig vergeben wurde, liegt womöglich daran, dass die hospitierte Fortbildungsmaßnahme primär auf die Vertiefung der fachlichen und fachdidaktischen Grundlagen zielte und weniger auf die Gestaltung inklusiver Settings. 
Die systematische Analyse des Datenmaterials ermöglichte eine Ausdifferenzierung der durch die Fortbildungsbeobachtung angestoßenen Reflexionen bezüglich einer eher normativen (retrospektiv hinsichtlich der hospitierten Maßnahme) oder einer eher prospektiven (hinsichtlich der zukünftigen Maßnahme) Dimension. Die normativen Reflexionen umfassten vor allem allgemeine Positionierungen oder konkrete Beobachtungen der Multiplikatorinnen, die sich auf die hospitierte Fortbildungsmaßnahme bezogen. Beide Reflexionsfokusse wiesen in der Regel einen wertenden Charakter - bestätigend/positiv oder herausfordernd/kritisch - auf. Die prospektiven Reflexionen enthielten bereits erste Adaptionsideen der Multiplikatorinnen, indem auf vorab existierende Einstellungen ebenso wie auf konkret hospitierte und reflektierte Herausforderungen konstruktiv reagiert wurde. Die von Sherin und Drake (2009) identifizierten Adaptionsprozesse omit, replace und create aufgreifend wurden die prospektiven Reflexionen der Multiplikatorinnen den Dimensionen erweiternd/additiv \& kreativ erneuernd, reduzierend/subtraktiv und alternativ/ ersetzend zugeordnet. Zum Teil ließen sich direkte Verbindungen zwischen den als herausfordernd/kritisch eingestuften normativen Reflexionen und den prospektiven Reflexionsdimensionen aufzeigen. Denkbar wären auch prospektive Reflexionen zu positiven Aspekten mit konkret formulierten Alternativen, die jedoch im hier ausgewerteten Material - bezogen auf die Teilnehmendenorientierung - nicht identifiziert werden konnten (vgl. Abschn. 5.1).

Das Gestaltungsprinzip der Teilnehmendenorientierung konnte bei der Analyse der moderierten Reflexionsgespräche mithilfe eines zunächst deduktiv entwickelten und im Verlauf des Analyseprozesses weiter verfeinerten Kategoriensystems differenzierter erfasst werden. Hierbei konnte herausgestellt werden, dass insbesondere der Schulkontext der Teilnehmenden (SK-T) (137 Codes) und ihr vorhandenes wie nicht vorhandenes fachliches und fachdidaktisches (Vor-)Wissen (KV-FFW-T-Prä) (43 Codes) im Reflexionsfokus der Multiplikatorinnen standen (vgl. Heinrich et al. 2013). Es lässt sich vermuten, dass die starke Fokussierung auf den Schulkontext der Teilnehmenden einerseits auf die vielschichtigen anzutreffenden Gestaltungen inklusiven Fachunterrichts und andererseits auf die Diskrepanz zum eigenen schulischen Kontext der Multiplikatorinnen zurückzuführen ist. Letzteres kann sowohl das aktuelle Tätigkeitsfeld der Multiplikatorinnen im Bereich der Aus- und Fortbildung betreffen als auch die eigenen Unterrichtserfahrungen. Der Fokus auf fachliches und fachdidaktisches (Vor-)Wissen liegt vermutlich in der Zielsetzung der Maßnahme begründet, den teilnehmenden Lehrpersonen ein flexibles, adaptives und fachlich sinnvolles Agieren im inklusiven Mathematikunterricht der Grundschule zu ermöglichen (vgl. Abschn. 3).

In den moderierten Reflexionsgesprächen bestätigten sich die bei der Analyse der schriftlichen Beobachtungsaufträge identifizierten Reflexionsdimensionen. Es ließen sich normative bestätigend/positive Äußerungen der Multiplikatorinnen hinsichtlich der benannten Aspekte finden. Der Großteil der Reflexionen war jedoch der Dimension herausfordernd/kritisch zuzuordnen: Die Multiplikatorinnen richteten ihren Blick allerdings deutlich häufiger auf die fachfremden Sonderpädagoginnen und Sonderpädagogen als eine Teilnehmendengruppe, die besondere Anforderungen an die Planung und Gestaltung von mathematikspezifischen Fortbildungen stellt, als dass sie die hospitierte Fortbildungsmaßnahme negativ bewerteten. Dies entspricht 
auch der Beobachtung, dass sich besonders viele normative bestätigend/positive Reflexionen in Bezug auf das in der Fortbildung vermittelte fachliche und fachdidaktische Wissen ( $K V$-FFW-T-Post) der Teilnehmenden finden ließen. Die übrigen Hauptkategorien der Teilnehmendenorientierung wurden deutlich seltener codiert (vgl. Tab. 5). Auch hier überwog in Bezug auf die normativen Reflexionen die herausfordernd/kritische Dimension. Eine direkte Verknüpfung zwischen normativer und prospektiver Reflexionsdimension - insbesondere normativ herausfordernd/ kritischer und prospektiv erweiternd/additiver \& kreativ/erneuernder Reflexionen konnte für etwa ein Zehntel der Reflexionen zum Gestaltungsprinzip der Teilnehmendenorientierung aufgezeigt werden. Prospektive Reflexionen der Dimensionen reduzierend/subtraktiv und alternativ/ersetzend wurden nur an sehr wenigen Stellen im Datenmaterial ausfindig gemacht.

Die identifizierten Reflexionsfokusse der Multiplikatorinnen decken sich mit dem in Abschn. 2.4 herausgestellten Spezifikum der Fachfremde sowie des besonderen schulischen Einsatzes der Sonderpädagoginnen und Sonderpädagogen. Reflexionen, die sich auf die sonderpädagogische Expertise der Teilnehmenden bezogen, fanden sich hingegen deutlich seltener. Ein möglicher Grund dafür könnte der fachliche und fachdidaktische Schwerpunkt der Fortbildung sein (s. oben). Zudem ist anzumerken, dass das sonderpädagogische Wissen der Teilnehmenden, von den Multiplikatorinnen nicht zwangsläufig als mit den Fortbildungsinhalten ,,kompatibel“ eingeschätzt wurde. Um die fachlich-fachdidaktische Expertise der Teilnehmenden stärker mit ihrer sonderpädagogischen Expertise (vgl. Moser und Kropp 2015) zu verknüpfen, könnten entsprechende Vorschläge in das Fortbildungsmaterial aufgenommen oder aber in zukünftigen moderierten Reflexionsgesprächen zum Gegenstand gemacht werden. Dass das Organisations- und Beratungswissen der Teilnehmenden ( $K V$ $O B W-T$ ) von den Multiplikatorinnen fast gänzlich unberücksichtigt blieb, ist möglicherweise darauf zurückzuführen, dass diese Wissenskomponente auch im Rahmen der hospitierten Fortbildungsmaßnahme nicht explizit angesprochen wurde.

Die Vielzahl der herausgestellten Reflexionen - insbesondere die Verknüpfung der normativen und der prospektiven Dimensionen - lässt darauf schließen, dass der Blick der Multiplikatorinnen für die spezifische Teilnehmendengruppe der fachfremd unterrichtenden Sonderpädagoginnen und Sonderpädagogen und damit auch für zentrale Elemente der Fortbildungsplanung und -durchführung für eben diese Teilnehmendengruppe geschärft wurden.

Eine Besonderheit der Studie stellte die während der ersten Phase von den Multiplikatorinnen eingenommene „Doppelrolle“ dar. Die Multiplikatorinnen setzten sich einerseits als Fortbildungsteilnehmende aktiv mit dem Fortbildungsgegenstand auseinander und erhielten andererseits die Möglichkeit, die Reaktionen der fachfremd unterrichtenden Sonderpädagoginnen und Sonderpädagogen auf den spezifischen Fortbildungsgegenstand und seine fortbildungsdidaktische wie -methodische Aufbereitung zu beobachten und zu reflektieren (vgl. Abb. 3, Abschn. 2.5).

Hinsichtlich der Beziehungen zwischen den Teilnehmenden und dem Fortbildungsgegenstand wurde deutlich, dass für die Multiplikatorinnen das didaktische Tetraeder der Unterrichtsebene - mitsamt seinem Kontext - zum Lerngegenstand der Fortbildungsebene wurde: Die Multiplikatorinnen hatten stets den Schulkontext der Teilnehmenden im Blick; insbesondere die Schülerinnen und Schüler der Son- 
derpädagoginnen und Sonderpädagogen sowie deren individuelle Bedarfe. Selbiges galt für die Funktionen und Möglichkeiten der Sonderpädagoginnen und Sonderpädagogen innerhalb des Unterrichts. Die Multiplikatorinnen waren darauf bedacht, den Teilnehmenden konkrete Unterrichtsmaterialien vorzustellen und deren Einsatzmöglichkeiten im inklusiven Mathematikunterricht mit den Teilnehmenden gemeinsam zu erarbeiten. Auch die Passung der fachlichen Fortbildungsinhalte und der individuellen Voraussetzungen und Bedarfe der Sonderpädagoginnen und Sonderpädagogen wurden von den Multiplikatorinnen reflektiert und diskutiert. Der zuerst genannte Aspekt zeigte sich vor allem in den normativen und prospektiven Reflexionen zum fachlichen und fachdidaktischen Vorwissen der Teilnehmenden und des - nach Ansicht der Multiplikatorinnen - noch zu erwerbenden fachspezifischen Wissens. Bezüglich des noch nicht vorhandenen Wissens der Teilnehmenden wurde eine starke Fokussierung auf den Bereich des theoretischen fachlichen und fachdidaktischen Wissens der Sonderpädagoginnen und Sonderpädagogen deutlich. Hier konnte unter anderem eine enge Verknüpfung der Gestaltungsprinzipien Teilnehmendenorientierung und Kompetenzorientierung herausgestellt werden, stellten doch der Auf- und Ausbau fachlicher und fachdidaktischer Kompetenzen das zentrale Ziel der Fortbildungsmaßnahme dar. Die Folge waren prospektive Reflexionen hinsichtlich der konkreten fachlichen und fachdidaktischen Fortbildungsinhalte sowie deren fortbildungsdidaktische Aufbereitung und methodische Einbettung (zum Beispiel Bereitstellung von Fachtexten über die Moodle-Plattform, Impulse zur Anregung fachlicher Diskussionen etc.). Im Rahmen weiterer Analysen kann genauer überprüft werden, in welchem Verhältnis das Gestaltungsprinzip der Teilnehmendenorientierung zu den übrigen DZLM-Gestaltungsprinzipien steht.

Die Beobachtung der Fortbildungsmaßnahme und die damit verbundenen konkreten Erfahrungen haben vielschichtige Reflexionen auf Seiten der Multiplikatorinnen angestoßen, deren tatsächliche Konsequenzen für die anschließende eigenständige Durchführung der Fortbildungsmaßnahme näher zu untersuchen sind (vgl. Phase 3, Abschn. 3). Einen Schwerpunkt der weiteren Datenauswertung wird daher der Abgleich des vom DZLM entwickelten mit dem von den Multiplikatorinnen adaptierten Fortbildungsmaterial bilden. Somit rückt der Tetraeder-Eckpunkt der Materialien und Medien (vgl. Abb. 2) stärker in den Fokus. Dabei soll untersucht werden, in welchem Verhältnis die vorgenommenen Adaptionen der Multiplikatorinnen zu den in der Reflexionsphase von ihnen getätigten normativen und prospektiven Reflexionen stehen (vgl. Ratte 2019); zum Beispiel: Welche prospektiven Reflexionen werden umgesetzt? Werden als kritisch bewertete Elemente der Fortbildung dennoch übernommen und falls ja, warum? Des Weiteren wird zu prüfen sein, ob die identifizierten normativen und prospektiven Reflexionen der Multiplikatorinnen eine mögliche Grundlage für die Entwicklung eines Reflexionsleitfadens bilden können, welcher anderen Multiplikatorinnen und Multiplikatoren zukünftig bei der Hospitation einer entsprechenden Fortbildungsmaßnahme zur Verfügung gestellt werden kann.

Die vorliegenden Ergebnisse weisen darauf hin, dass das hier zugrundeliegende Qualifizierungsmodell, das die gemeinsame Hospitation und Reflexion der Multiplikatorinnen verknüpft, ein tragfähiges Modell zur Professionalisierung von Multiplikatorinnen und Multiplikatoren sein kann (zu anderen Modellen vgl. z. B. Was- 
song 2017; Schuler und Wittmann 2018; Leufer et al. 2019). Die zum Abschluss der Studie durchgeführten Interviews (vgl. Phase 3, Abschn. 3) sollen Einblicke dahingehend geben, inwieweit die Multiplikatorinnen und Multiplikatoren diesen Perspektivwechsel als hilfreich empfunden haben.

\section{Literatur}

Barzel, B., \& Selter, C. (2015). Die DZLM-Gestaltungsprinzipien für Fortbildungen. Journal für Mathematik-Didaktik, 36(2), 259-284.

Barzel, B., Biehler, R., Blömeke, S., Brandtner, R., Bruns, J., Dohrmann, C., Kortenkamp, U., Lange, T., Leuders, T., Rösken-Winter, B., Scherer, P., \& Selter, C. (2018). Das Deutsche Zentrum für Lehrerbildung Mathematik - DZLM. In R. Biehler, T. Lange, T. Leuders, B. Rösken-Winter, P. Scherer \& C. Selter (Hrsg.), Mathematikfortbildungen professionalisieren: Konzepte, Beispiele und Erfahrungen des Deutschen Zentrums für Lehrerbildung Mathematik (S. 7-39). Wiesbaden: Springer Spektrum.

Baumert, J., \& Kunter, M. (2006). Stichwort: Professionelle Kompetenz von Lehrkräften. Zeitschrift für Erziehungswissenschaft, 9(4), 469-520.

Bennewitz, H. (2012). Der Blick auf Lehrer/-innen. In H. de Boer \& S. Reh (Hrsg.), Beobachtung in der Schule - Beobachten lernen (S. 203-314). Wiesbaden: VS.

Berndt, C., Häcker, Th , \& Leonard, T. (2017). Reflexive Lehrerbildung revisited. Traditionen - Zugänge Perspektiven. Bad Heilbrunn: Klinkhardt.

de Boer, H. (2012). Beobachtung und Professionalisierung. In H. de Boer \& S. Reh (Hrsg.), Beobachtung in der Schule - Beobachten lernen (S. 201-311). Wiesbaden: VS.

Borasi, R., \& Fonzi, J. (2002). Professional Development that Supports School Mathematics Reform. Arlington: National Science Foundation.

Borko, H., Koellner, K., \& Jacobs, J. (2014). Examining novice teacher leaders' facilitation of mathematics professional development. Journal of Mathematical Behavior, 33, 149-167.

Buhren, C.G. (2012). Kollegiale Hospitation: Verfahren, Methoden und Beispiele aus der Praxis. Köln: Carl Link.

Carpenter, T.P., \& Fennema, E. (1992). Cognitively guided instruction: building on the knowledge of students and teachers. International Journal of Research in Education, 17(5), 457-470.

Cendon, E. (2017). Reflexion in der Hochschulweiterbildung. Verbindungsglied zwischen unterschiedlichen Erfahrungswelten. Zeitschrift Hochschule und Weiterbildung. https://doi.org/10.4119/zhwb212.

Clarke, D. M. (1994). Ten key principles from research for the professional development of mathematics teachers. In D. B. Aichele \& A. F. Croxford (Hrsg.), Professional development for teachers of mathematics (S. 37-48). Reston: NCTM.

Dalehefte, I. M., \& Kobarg, M. (2013). Aus Unterrichtsbeobachtungen lernen. Handreichung des Programms SINUS an Grundschulen. Kiel: IPN-Materialien. http://www.sinus-an-grundschulen.de/ fileadmin/uploads/Material_aus_SGS/Handreichung_DalehefteKobarg_fuer_Web.pdf. Zugegriffen: 18. Apr. 2019.

Dengerink, J., Lunenberg, M., \& Korthagen, F. (2015). The professional teacher educator: six roles. Beiträge zur Lehrerinnen- und Lehrerbildung, 33(3), 334-344.

Dewey, J. (2000). Demokratie und Erziehung: Eine Einleitung in die philosophische Pädagogik; mit einer umfangreichen Auswahlbibliographie. Weinheim: Beltz.

Dewey, J. (2002). Wie wir denken. Zürich: Verlag Pestalozzianum.

DZLM (2015). Qualifizierung von Multiplikatorinnen und Multiplikatoren. Version: 07.03.2015. https:// dzlm.de/files/uploads/DZLM-2.0-Konzept\%20Multiplikatoren-20150316_FINAL.pdf. Zugegriffen: 20. Febr. 2020.

Franke, M.L., Carpenter, T. P., Levi, L., \& Fennema, E. (2001). Capturing teachers' generative change: a follow-up study of professional development in mathematics. American Educational Research Journal, 38, 635-689.

Gasterstädt, J., \& Urban, M. (2016). Einstellung zu Inklusion? Implikationen aus Sicht qualitativer Forschung im Kontext der Entwicklung inklusiver Schulen. Empirische Sonderpädagogik, (1), 54-66.

Heinrich, M., Urban, M., \& Werning, R. (2013). Grundlagen, Handlungsstrategien und Forschungsperspektiven für die Ausbildung und Professionalisierung von Fachkräften für inklusive Schulen. In 
H. Döbert \& H. Weishaupt (Hrsg.), Inklusive Bildung professionell gestalten - Situationsanalyse und Handlungsempfehlungen (S. 69-133). Münster: Waxmann.

Heinrich, M., \& Werning, R. (2013). „It's Team-Time“? Unterrichtskooperation von SonderpädagogInnen und Fachlehrkräften angesichts zeitlich knapper Ressourcen und asymmetrischer Beziehungen. Journal für Schulentwicklung, (4), 26-32.

Herzog, W. (1995). Reflexive Praktika in der Lehrerinnen- und Lehrerbildung. Beiträge zur Lehrerbildung, 13(3), 253-273.

Korff, N. (2015). Inklusiver Mathematikunterricht in der Primarstufe: Erfahrungen, Perspektiven und Herausforderungen. Hohengehren: Schneider.

Krainer, K. (1998). Some considerations on problems and perspectives of mathematics teacher in-service education. In C. Alsina, J. M. Alvarez, B. Hodgson, C. Laborde \& A. Perez (Hrsg.), The 8th International Congress on Mathematical Education (ICME 8) (S. 303-321). Sevilla: S.A.E.M. Thales.

Kuckartz, U. (2016). Qualitative Inhaltsanalyse: Methoden, Praxis, Computerunterstützung. Weinheim Basel: Beltz Juventa.

Leinhardt, G., Putnam, R. T., Stein, M.K., \& Baxter, J. (1991). Where subject knowledge matters. In J.E. Brophy (Hrsg.), Advances in research on teaching (Bd. 2, S. 87-113). Greenwich: JAI Press.

Lencer, S., \& Strauch, A. (2016). Das GRETA-Kompetenzmodell für Lehrende in der Erwachsenen- und Weiterbildung. https://www.die-bonn.de/doks/2016-erwachsenenbildung-02.pdf. Zugegriffen: 20. Febr. 2020.

Leufer, N., Prediger, S., Mahns, P., \& Kortenkamp, U. (2019). Facilitators' adaptation practices of curriculum material resources for professional development courses. International Journal of STEM Education, 6, 1-18. https://doi.org/10.1186/s40594-019-0177-0.

Lipowsky, F. (2010). Lernen im Beruf - Empirische Befunde zur Wirksamkeit von Lehrerfortbildung. In F. H. Müller, A. Eichenberger, M. Lüders \& J. Mayr (Hrsg.), Lehrerinnen und Lehrer lernen. Konzepte und Befunde zur Lehrerfortbildung (S. 51-70). Münster: Waxmann.

Lipowsky, F. (2014). Theoretische Perspektiven und empirische Befunde zur Wirksamkeit von Lehrerfortund Weiterbildung. In E. Terhart, H. Bennewitz \& M. Rothland (Hrsg.), Handbuch der Forschung zum Lehrerberuf (S. 511-541). Münster: Waxmann.

Lipowsky, F., \& Rzejak, D. (2017). Fortbildungen für Lehrkräfte wirksam gestalten - Erfolgsversprechende Wege und Konzepte aus Sicht der empirischen Bildungsforschung. Bildung und Erziehung, 70(4), 379-399.

Luft, J.A., \& Hewson, P.W. (2014). Research on teacher professional development in science. In S. K. Abell \& N. G. Lederman (Hrsg.), Handbook of research in science education (Bd. 2, S. 889-909). New York: Routledge.

Ma, L. (1999). Knowing and teaching elementary mathematics. Teachers' understanding of fundamental mathematics in China and the United States. Mahwah: Routledge.

Moser Opitz, E. (2005). Lernschwierigkeiten Mathematik in Klasse 5 und 8. Eine empirische Untersuchung zu fehlenden mathematischen Basiskompetenzen. Vierteljahresschrift für Heilpädagogik und ihre Nachbargebiete, 74(2), 113-128.

Moser Opitz, E., \& Nührenbörger, M. (2015). Diagnostik und Leistungsbeurteilung. In R. Bruder, L. Hefendehl-Hebeker, B. Schmidt-Thieme \& H.-G. Weigand (Hrsg.), Handbuch der Mathematikdidaktik (S. 491-512). Wiesbaden: Springer Spektrum.

Moser, V., \& Kropp, A. (2015). Kompetenzen in Inklusiven Settings (KIS) - Vorarbeiten zu einem Kompetenzstrukturmodell sonderpädagogischer Lehrkräfte. In Th Häcker \& M. Walm (Hrsg.), Inklusion als Entwicklung - Konsequenzen für Schule und Lehrerbildung (S. 185-212). Bad Heilbrunn: Klinkhardt.

MSW (2008). Richtlinien und Lehrpläne für die Grundschule in Nordrhein-Westfalen. Frechen: Ritterbach.

Neuweg, H.G. (2005). Emergenzbedingungen pädagogischer Könnerschaft. In H. Heid \& C. Harteis (Hrsg.), Verwertbarkeit. Ein Qualitätskriterium (erziehungs-)wissenschaftlichen Wissens? (S. 205-228). Wiesbaden: VS.

Nührenbörger, M., \& Steinbring, H. (2009). Forms of mathematical interaction in different social settings examples from students', teachers' and teacher-students' communication about mathematics. Journal of Mathematics Teacher Education, 12(2), 111-132.

Peters-Dasdemir, J., Holzäpfel, L., Barzel, B., \& Leuders, T. (2020). Professionalization of Facilitators in Mathematics Education: A Competency Framework. In M. Inprasitha, N. Changsri \& N. Boonsena (Hrsg), Proceedings of the 44th Conference of the International Group for the Psychology of Mathematics Education, Interim Vol. (S. 457-465). Khon Kaen, Thailand: PME.

Prediger, S., Leuders, T., \& Rösken-Winter, B. (2017). Drei-Tetraeder-Modell der gegenstandsbezogenen Professionalisierungsforschung. Fachspezifische Verknüpfung von Design und Forschung. In K. Zie- 
rer (Hrsg.), Jahrbuch für Allgemeine Didaktik 2017. Thementeil: Allgemeine Didaktik und Lehrer/ innenbildung (S. 159-177). Baltmannsweiler: Schneider Hohengehren.

Ratte, L. (2019). Reflexive Adaptionsprozesse von MultiplikatorInnen beim Design einer Fortbildungsmaßnahme zum inklusiven Mathematikunterricht. In A. Frank, S. Krauss \& K. Binder (Hrsg.), Beiträge zum Mathematikunterricht 2019 (S. 959-962). Münster: WTM.

Reusser, K., \& Tremp, P. (2008). Diskussionsfeld „Berufliche Weiterbildung von Lehrpersonen“. Beiträge zur Lehrerinnen- und Lehrerbildung, 26(1), 5-10.

Roesken-Winter, B., Schüler, S., Stahnke, R., \& Blömeke, S. (2015). Effective CPD on a large scale: examining the development of multipliers. ZDM - The International Journal on Mathematics Education, 47(1), 1-12.

Rösike, K., Prediger, S., \& Barzel, B. (2016). DZLM-Gestaltungsprinzipien für Fortbildungen von Lehrpersonen. Eine Handreichung zur Konkretisierung der Prinzipien. https://dzlm.de/sites/dzlmtheme/ files/DZLM-Gestaltungsprinzipien-Konkretisierung_161201_0.pdf. Zugegriffen: 20. Febr. 2020.

Rösken-Winter, B., \& Kramer, J. (2013). Lehrerfortbildungen als berufsbegleitende Erwachsenenbildung: Einfluss von Vorwissen und Auswirkungen auf die Praxis. In Beiträge zum Mathematikunterricht 2013 (S. 842-845). Münster: WTM.

Rzejak, D., Künsting, J., Lipowsky, F., Fischer, E., Dezhgahi, U., \& Reichardt, A. (2014). Facetten der Lehrerfortbildungsmotivation. Eine faktorenanalytische Betrachtung. Journal for educational research online, 6(1), 139-159.

Sato, M., Akita, K., \& Iwakawa, N. (1993). Practical thinking styles of teachers: a comparative study of expert and novice thought processes and its implications for rethinking teacher education in Japan. Peabody Journal of Education, 68(4), 100-110.

Scherer, P. (2019). Inklusiver Mathematikunterricht - Herausforderungen bei der Gestaltung von Lehrerfortbildungen. In A. Büchter, M. Glade, R. Herold-Blasius, M. Klinger, F. Schacht \& P. Scherer (Hrsg.), Vielfältige Zugänge zum Mathematikunterricht - Konzepte und Beispiele aus Forschung und Praxis (S. 327-340). Wiesbaden: Springer.

Scherer, P., \& Steinbring, H. (2006). Noticing children's learning processes-teachers jointly reflect on their own classroom interaction for improving mathematics teaching. Journal of Mathematics Teacher Education, 9(2), 157-185.

Scherer, P., Nührenbörger, M., \& Ratte, L. (2019). Inclusive mathematics - in-service training for outof-field teachers. In J. Novotná \& H. Moraova (Hrsg.), SEMT 2019. Proceedings: opportunities in learning and teaching elementary mathematics (S. 382-391). Prague: Charles University, Faculty of Education.

Schön, D. A. (1983). The reflective practitioner. London: Temple Smith.

Schön, D. A. (1988). Educating the reflective practitioner. San Francisco: Jossey-Bass.

Schuler, S., \& Wittmann, G. (2018). Empirische Befunde zur beruflichen Situation von 5 Multiplikatorinnen und Multiplikatoren für den Mathematikunterricht an Grundschulen. In R. Biehler, T. Lange, T. Leuders, B. Rösken-Winter, P. Scherer \& C. Selter (Hrsg.), Mathematikfortbildungen professionalisieren - Konzepte, Beispiele und Erfahrungen des Deutschen Zentrums für Lehrerbildung Mathematik (S. 79-98). Wiesbaden: Springer.

Sherin, M.G., \& Drake, C. (2009). Curriculum strategy framework: investigating patterns in teachers' use of a reform-based elementary mathematics curriculum. Journal of Curriculum Studies, 41(4), 467-500.

Timperley, H., Wilson, A., Barrar, H., \& Fung, I. (2007). Teacher professional learning and development. Best Evidence Synthesis Iteration. Wellington: New Zealand: Ministry of Education.

Wassong, T. (2017). Datenanalyse in der Sekundarstufe I als Fortbildungsthema. Theoriegeleitete Konzeption und Evaluation einer Multiplikatorenqualifizierung. Wiesbaden: Springer.

Wember, F. B. (1998). Zweimal Dialektik: Diagnose und Intervention, Wissen und Intuition. Sonderpädagogik, 28(2), 106-120.

Wilson, S. M., \& Berne, J. (1999). Teacher learning and the acquisition of professional knowledge: an examination of research on contemporary professional development. Review of Research in Education, 24(1), 173-209.

Wyss, C. (2008). Zur Reflexionsfähigkeit und -praxis der Lehrperson. bildungsforschung, 5(2), 1-15.

Wyss, C. (2013). Unterricht und Reflexion. Eine mehrperspektivische Untersuchung der Unterrichts- und Reflexionskompetenz von Lehrkräften. Münster: Waxmann.

Zaslavsky, O. (2008). Meeting the challenges of mathematics teacher education through design and use of tasks that facilitate teacher learning. In B. Jaworski \& T. Wood (Hrsg.), The mathematics teacher educator as a developing professional (Bd. 4, S. 93-114). Rotterdam: Sense Publishers. 